\title{
ADVANCES IN MEASUREMENTS OF PHYSICAL PARAMETERS OF SEMICONDUCTOR LASERS
}

\author{
G. E. SHTENGEL \\ Lucent Technologies Bell Laboratories, 9999 Hamilton Blvd., Breinigsville, PA 18031 \\ USA \\ G. L. BELENKY \\ Department of Electrical Engineering, SUNY at Stony Brook, Stony Brook, NY 11794 \\ USA \\ Lucent Technologies Bell Laboratories, 700 Mountain Ave., Murray Hill, NJ 07974 USA \\ M. S. HYBERTSEN \\ Lucent Technologies Bell Laboratories, 700 Mountain Ave., Murray Hill, NJ 07974 USA \\ R. F. KAZARINOV \\ Lucent Technologies Bell Laboratories, 9999 Hamilton Blvd., Breinigsville, PA 18031 \\ USA \\ D. A. ACKERMAN \\ Lucent Technologies Bell Laboratories, 700 Mountain Ave., Murray Hill, NJ 07974 USA
}

We present a summary of advances in characterization techniques allowing for comprehensive study of physical processes in semiconductor lasers.

\section{Outline}

1. Introduction.

2. Measurements of the optical gain.

2.1. Determination of the optical gain from the amplified spontaneous emission.

2.2. Determination of the optical gain from the true spontaneous emission.

2.3. Determination of the gain in broad-area lasers.

3. Measurement of the optical loss.

4. Measurement of the carrier leakage in semiconductor heterolasers.

4.1. Optical technique of studying the carrier leakage.

4.2. Electrical technique of studying the carrier leakage.

5. Electrical and optical measurements of RF modulation response below threshold.

5.1. Determination of the differential carrier lifetime from the device impedance.

5.2. Determination of the differential carrier lifetime from the optical response measurements.

6. Optical measurements of RF modulation response and RIN above threshold

6.1. Determination of the resonant frequency and damping factor using carrier subtraction technique. Determination of the differential gain and the gain compression coefficient.

6.2. Determination of the resonant frequency and differential gain from the RIN measurements.

7. Measurements of the linewidth enhancement factor.

7.1. Measurements of linewidth enhancement factorfrom ASE and TSE spectra.

8. Measurements of the carrier temperature and carrier heating in semiconductor lasers.

8.1. Determination of carrier heating from wavelength chirp and TSE measurements.

9. References. 


\section{Introduction}

The fast growing use of semiconductor lasers in various fields including fiber communication systems, optical data storage, remote sensing etc. places very stringent requirements on device performance. This requires a detailed understanding of physical processes governing the behavior of laser diodes. A semiconductor laser fundamentally operates by electrical transport of carriers to a central active layer of the device where they recombine to produce optical gain and support stimulated emission of photons. However, in modern, optimized devices, a non-trivial cascade of microscopic processes is involved.

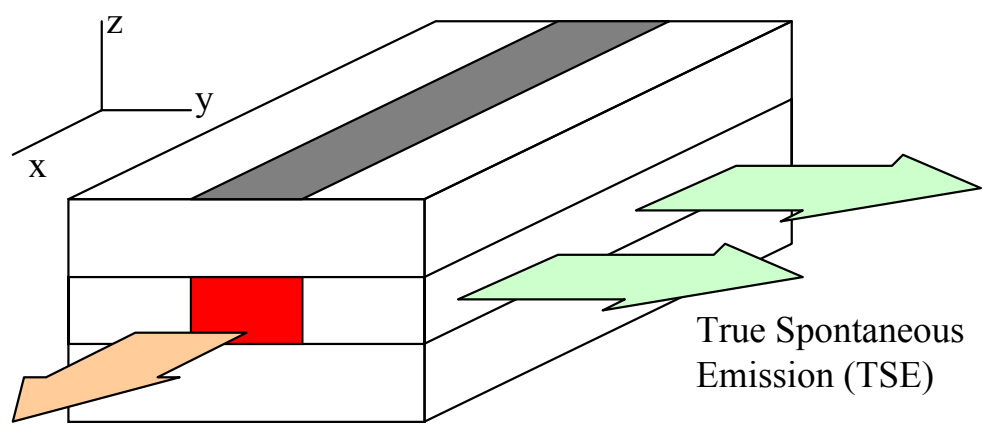

Amplified Spontaneous Emission (ASE) + Stimulated Emission

Fig. 1.1. Simplified scheme of the laser diode.

Most of the experimental techniques presented here are developed in relation to telecommunication lasers. These lasers are usually designed so the output radiation can be coupled into the single-mode optical fiber. Therefore the lasers themselves usually employ single waveguide mode design. Two common approaches are illustrated in Fig. 1.1. The active layer, which may be either bulk material or multiple quantum wells, is confined in the z-direction by the cladding layers of lower refractive index, forming an optical waveguide in this direction. In the y-direction the confinement is obtained by either etch and regrowth with material of lower index and lower conductivity (index guided buried heterostructure $(\mathrm{BH})$ ), or partial etch down to the cladding layer, forming a ridge waveguide laser. The laser resonator is usually obtained by cleaving the structure along the crystal axes. Cleaved facets form two mirrors of the laser cavity along the xdirection. Light emerging from these two facets travels along the laser cavity and is therefore affected by the presence of the optical gain or loss. At currents higher than threshold it is predominantly stimulated emission, below threshold it is amplified spontaneous emission (ASE). The light emerging from the side of a chip (y- or $\mathrm{z}$ direction) experiences almost no amplification or absorption, because it travels only a very short distance inside the active layer media, and there is neither waveguide nor resonator in this direction. This is true spontaneous emission (TSE), almost completely unaffected by the geometry of the laser structure.

Study of this light emission offers tremendous insight to the operation of the laser, including such fundamental aspects as the optical gain and loss. However, optimization 
of the properties of the laser requires understanding the connection between the optical emission and the carrier transport processes in the device. Figure 1.2 illustrates this point for a multiple quantum well buried heterostructure laser. The active layer at the core of the diode is also at the center of a diode, which is operated in forward bias resulting in the injection of electrons and holes. The regions of material regrown along the edges of the active layer must be designed to minimize current flow around the active layer. Details of the carrier transport can have significant impact on steady state characteristics such as efficiency as well as the modulation response of the device. Within the active layer itself, electrons and holes are injected with higher energy due to the band offset at the heterojunction. Then the carriers must be captured into the quantum wells. There are different time scales associated to the carrier thermalization, cooling and capture. Furthermore, with multiple wells, the transport of carriers to populate wells further along the stack can involve several steps of capture and reemission. In general, the population of carriers above the barrier will be out of equilibrium with the populations bound in the wells. As the net stimulated emission rate rises, these populations will shift to support the required current flow. The light emission from the active region is determined by the arrangement of the carriers at each bias of the laser diode. The other carrier recombination processes, such as Auger, also depend on the details of the carrier distribution.
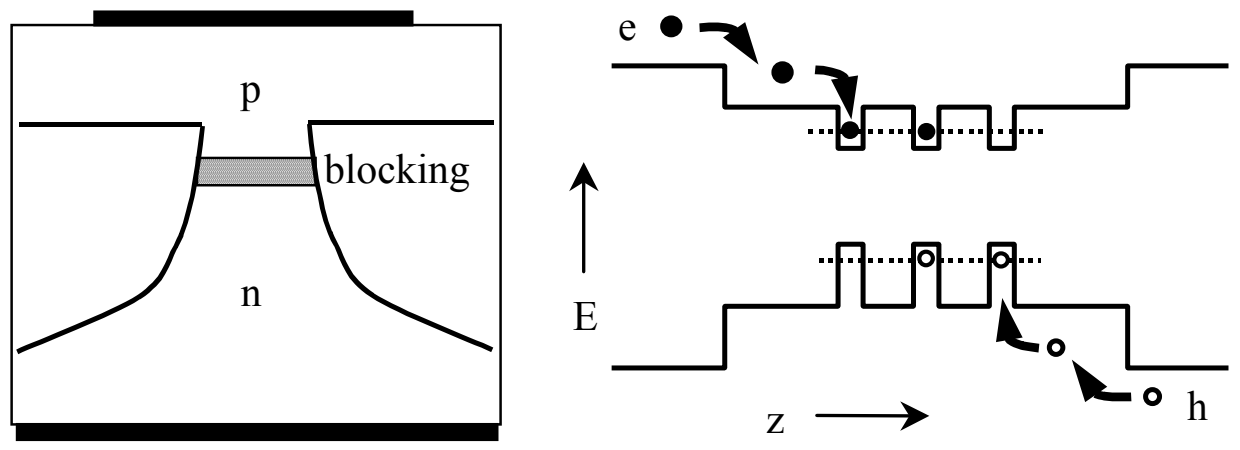

Fig. 1.2. Schematic cross section of a BH laser (left) and an illustration of some of the transport processes in the multiple quantum well active region (right).

A full experimental characterization of all of the processes outlined would be very challenging indeed. In this review, a broad set of electrical and optical techniques is described which give complimentary information on the operation of the laser diode. In general, the carrier distribution in the active layer strongly depends on drive current below threshold, but not above threshold. Physical processes below threshold are critical in determining the operating point of the laser. Therefore studying the electrical characteristics and optical emission below threshold is often more informative in the process of understanding the device performance. Some other parameters, such as leakage current or wavelength chirp can only be deduced from above threshold measurements. Taken together, these measurements provide critical experimental feedback in the process of laser diode optimization. They are also essential information to guide our understanding of the microscopic physical processes described above and efforts to simulate those processes. 


\section{Measurements of the optical gain}

One of the most important parameters relating the physical properties of the semiconductor structure to output characteristics of the laser diode is the optical gain. Optical gain and its dependence on the operating conditions determine not only the basic output characteristics, such as threshold current, but also the temperature dependence of the output characteristics, as well high-speed performance of the laser. Various techniques for the measurement of the optical gain are described in this section. Their respective advantages and shortcomings are discussed on the basis of the presented experimental results for $1.3 \mu \mathrm{m}$ InGaAsP based lasers. Closely coupled to the optical gain measurements are the measurements of the optical loss, which is also a very important parameter. These are reviewed in Section 3.

The electric wave in the resonator of semiconductor can be written as:

$$
\vec{E}=\vec{E}_{0}(y, z) \cdot \exp (j(k x-\omega t))
$$

where $\vec{E}_{0}(y, z)$ is the magnitude of the field, and the complex propagation constant $k$ is:

$$
k(\lambda)=k^{\prime}+j k^{\prime \prime}=\frac{2 \pi N_{e f f}(\lambda)}{\lambda}-\frac{j}{2} g(\lambda)
$$

$N_{\text {eff }}$ is an effective index of refraction for the optical mode, and $g$ is the modal optical gain. The factor of 2 comes in because the optical gain is usually defined with respect to optical power, not optical field intensity. Modal optical gain $g(\lambda)$ is related to the material optical gain $G(\lambda)$ :

$$
g(\lambda)=\Gamma G(\lambda)-\alpha_{t o t}
$$

where $\Gamma$ is optical confinement factor (the fraction of the transverse optical mode overlapping the active layer and therefore experiencing optical gain) [1]. The total optical loss $\alpha_{t o t}$ consists of mirror loss and internal loss, usually attributed to free-carrier absorption and scattering from waveguide imperfections:

$$
\alpha_{\text {tot }}=\alpha_{\text {mirr }}+\alpha_{\mathrm{int}}=\frac{1}{L} \ln \left(\frac{1}{R}\right)+\alpha_{\mathrm{int}}
$$

The representation of the mirror loss as $1 / L \ln (1 / R)$ is valid only Fabry-Perot Lasers, $R=\sqrt{R_{1} \cdot R_{2}}$ being the geometrical mean of the mirror reflectivities, $L$ the laser resonator length.

It should be pointed out that the optical gain is defined in Eq. (2.2) as (in terms of variations):

$$
\delta g=-2 \delta k^{\prime \prime}=-\frac{4 \pi}{\lambda} \Gamma \delta N^{\prime \prime}
$$

In the literature [2] (Chapter 5.3.3 p.209), and [3] (Chapter.15.6, p.582-583) authors use the opposite sign in the Eq. (2.1) which leads to the opposite sign in the relation between the gain and the imaginary part of the refractive index. It will also lead to an opposite sign in the definition of the linewidth enhancement factor, as it will be discussed later. In our treatment we follow definitions of C. Henry [4], (p.4459). This difference is, of course, only in definitions and not in final results. 
Optical gain can be extracted from both ASE and TSE, as well as using some other methods, such as direct transmission measurement or variable stripe length (VSL) technique [5]. Direct transmission measurement may be advantageous when both facets are anti-reflection AR coated (mirror loss is very high) and Fabry-Perot contrast is very small. VSL technique has an advantage of not being influenced by the resonator quality. However it requires a special sample preparation and cannot be used for a single device. Only the methods based on ASE and TSE measurements will be discussed here.

\subsection{Determination of the optical gain from the amplified spontaneous emission}

In this section the gain measurements based on the analysis of the ASE spectra will be discussed. Fig. 2.1 shows the spectra of ASE in TE polarization for the $1.3 \mu \mathrm{m}$ BH laser [6] with a bulk active region and uncoated mirror facets. There can be seen a very clean interference pattern of the Fabry-Perot resonator formed by the cleaved mirrors. This interference pattern is used to analyze the optical gain.
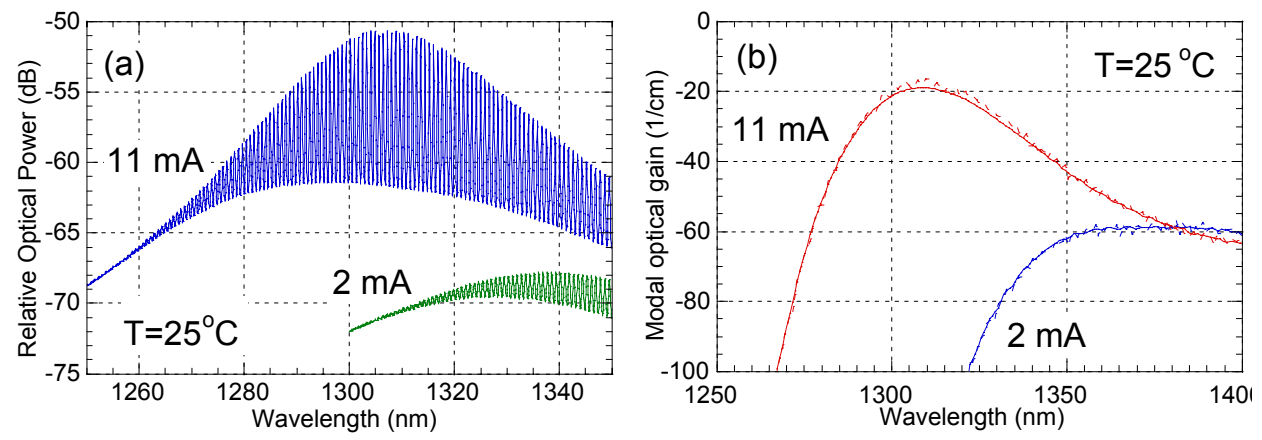

Fig. 2.1. (a) The ASE spectra at room temperature for different currents and (b) modal optical gain spectra extracted from these spectra using the Hakki-Paoli technique (solid line) and the Cassidy method (dashed line).

Following equation describes the dependence of the ASE intensity on the wavelength [7] in the approximation of uniform gain inside the laser cavity:

$$
I(\lambda)=\frac{B\left(1+R \cdot e^{\left(\Gamma G-\alpha_{\text {int }}\right) L}\right)(1-R)}{\left(1+R \cdot e^{\left(\Gamma G-\alpha_{\text {int }}\right) L}\right)^{2}-4 R \cdot e^{\left(\Gamma G-\alpha_{\text {int }}\right) L} \cdot \sin ^{2}\left(\frac{2 \pi L n}{\lambda}\right)}
$$

where $B$ is the proportionality coefficient equal to the total amount of ASE coupled into the lasing mode.

B. Hakki and T. Paoli proposed to determine the modal optical gain from the contrast of the ASE spectra [8]. Eq. (2.6) is used to obtain:

$$
g(\lambda)=\frac{1}{L} \ln \frac{\sqrt{r(\lambda)}-1}{\sqrt{r(\lambda)}+1}
$$

where $r(\lambda)$ is the peak-to-valley ratio:

$$
r(\lambda)=\frac{I_{\max }(\lambda)}{I_{\min }(\lambda)}
$$


D. Cassidy [9] proposed another way to analyze ASE in order to determine the material gain values. This technique, also called the mode-sum method, is based on a slightly different analysis of the same basic Eq. (2.6). E. Gordon demonstrated [7] that the optical gain in the oscillator system governed by Eq. (2.6) is:

$$
g(\lambda)=\frac{1}{L} \ln \frac{p(\lambda)-1}{p(\lambda)+1}
$$

where $p$ is the ratio of the integral over one mode to the mode minimum:

$$
p(\lambda)=\frac{\int_{\lambda_{1}}^{\lambda_{2}} I\left(\lambda^{\prime}\right) d \lambda^{\prime}}{I_{\min }(\lambda) \cdot\left(\lambda_{2}-\lambda_{1}\right)}
$$

D. Cassidy [9] and later L. Lam Sin Cho [10] have shown theoretically and experimentally that the mode-sum method is less sensitive to the spectral bandwidth of the measurement system than the Hakki-Paoli technique.

The modal optical gain extracted from the ASE spectra, shown in the Fig. 2.1a using the Hakki-Paoli and Cassidy techniques is shown in the Fig. 2.1b. Along with the conclusion that two methods give very similar results, there can be noticed some important differences. First, in the high gain regime (11 mA, around the gain maximum) the Hakki-Paoli technique slightly underestimates (by about $5 \mathrm{~cm}^{-1}$ ) the value of modal optical gain. As it was discussed above, this happens because the spectral resolution of the optical spectrum analyzer used in the experiment (HP70951B - 1尺) is not adequate to resolve appropriately the maxima in the ASE spectrum. Secondly, the Cassidy technique has higher noise due to inaccuracy in the detection of the wavelength of the mode minima.

The optical gain measurement is the essential part of several optical loss measurement techniques [11], as it is shown in the Section 3. Optical gain measurements combined with the differential carrier lifetime measurements [12] allow for analysis of the respective contributions of different physical mechanisms to a temperature dependence of the threshold current of $1.3 \mu \mathrm{m}$ lasers [13]. A similar set of measurements was also very useful for optimization of the high-speed performance of $1.55 \mu \mathrm{m}$ lasers [14], which will be discussed in the Section 7. Combinations of Hakki-Paoli and Cassidy methods were used to study the optical gain in lasers based on various material systems and designs in the entire wavelength range from visible [15] to far IR [16].

\subsection{Determination of the optical gain from the true spontaneous emission}

An alternative way of determining the optical gain was first proposed by V. Gribkovskii and V. Samoilyukovich [17] and has been extended for many applications by C. Henry et al [18]. It was later used by P. Blood et al [19] as a part of simultaneous analysis of optical gain and recombination based on TSE measurements.

This method is based on the general relations between the rates of spontaneous emission, stimulated emission, and optical absorption. It was shown in [18], that if carriers have Fermi-like distribution functions, the material optical gain is related to the absorption coefficient in the following way: 


$$
G(\varepsilon, \mu, T)=a(\mu, \varepsilon) \cdot\left\{\exp \left(\frac{\mu-\varepsilon}{k T}\right)-1\right\}
$$

where $\varepsilon$ is photon energy, $\mu$ the separation between the quasi Fermi levels of electrons and holes, and $\alpha(\mu, \varepsilon)$ the absorption coefficient of the material of the active layer. Generally, $\alpha(\mu, \varepsilon)$ itself depends on the band filling and, therefore, on $\mu$. Another important equation relates the intensity of spontaneous emission $I_{s p}(\varepsilon)$ to the absorption coefficient:

$$
I_{s p} \propto \varepsilon^{2} \cdot a(\varepsilon, \mu) \cdot \exp \left(\frac{\mu-\varepsilon}{k T}\right)
$$

where the proportionality sign allows for some constant factors to be omitted. They are of no importance because it is practically impossible to quantify the absolute measurements of the spontaneous emission. Combining Eq. (2.11)-(2.12):

$$
G(\varepsilon, \mu, T) \propto \frac{I_{s p}(\varepsilon)}{\varepsilon^{2}} \cdot\left\{1-\exp \left(\frac{\varepsilon-\mu}{k T}\right)\right\}
$$

In order to obtain the value of the material optical gain using Eq. (2.13) the value of the quasi-Fermi level separation, $\mu$, should be known. In [18], the authors have proposed utilizing the property of the Fabry-Perot lasers to lase at the wavelength where the material gain is maximum. Therefore its first derivative with respect to wavelength is zero, allowing a determination of the value of the quasi Fermi level separation at threshold. In order to determine this value at lower currents, authors of [18] have proposed to use the fact that at the high energies the absorption coefficient $\alpha(\mu, \varepsilon)$ is independent of the injection level. This allows for determination of the value of the quasi Fermi level separation below threshold. P. Rees and P. Blood have pointed out that this procedure can possibly lead to a serious error, since the Fermi-level separation has to be determined with very high accuracy in order for accurate calculation of the optical gain from the TSE spectra $[19,20]$. They have also pointed out that the carrier temperature enters the Eq. (2.13), which should be taken into account, since in the case of carrier heating the carrier temperature is not equal to the lattice temperature.

One more condition is required in order to use the Eq. (2.13) to determine quantitatively the gain spectra from the TSE spectra. Eq. (2.13) gives the value of the material optical gain in arbitrary units and a proper scaling factor needs to be determined in order to find the material or modal gain. C. Henry used the fact that a FP laser at threshold has a maximum gain equal to the total loss. If the laser has uncoated facets, the mirror loss can be easily calculated. Then the value of total loss can be estimated from $L$ $I$ curve slope with an assumption that the internal efficiency is $100 \%$. This approximation is not accurate in many cases, however, and this procedure cannot be used for the lasers with coated mirrors or lasers with wavelength-selective feedback, such as DFB or DBR lasers. P. Blood has proposed to use the known value of absorption coefficient above the absorption edge [19].

The values of the total loss and quasi Fermi level separation can also be determined using the transparency measurements described in the following section. The TSE spectra, recorded from the side of an uncoated DFB laser at different currents are presented in Fig. 2.2a. Noticeably, there are no Fabry-Perot ripples in the TSE spectra. The TSE spectrum is also much broader and extends much further into high energies 
(lower wavelengths) than the ASE, because the TSE is not affected by reabsorbtion in the active layer. The modal optical gain was determined from these spectra using the values of the total loss and the transparency energy, obtained using the zero-contrast method, described in the Section 3. The modal optical gain spectra are plotted in Fig. 2.2b together with the spectra extracted from the ASE as described in the Section 1. The gain spectrum extracted from the TSE is much wider than shown in Fig. 2.2b, where the narrow wavelength range is chosen for the sake of comparing it to the gain spectrum extracted from the ASE.
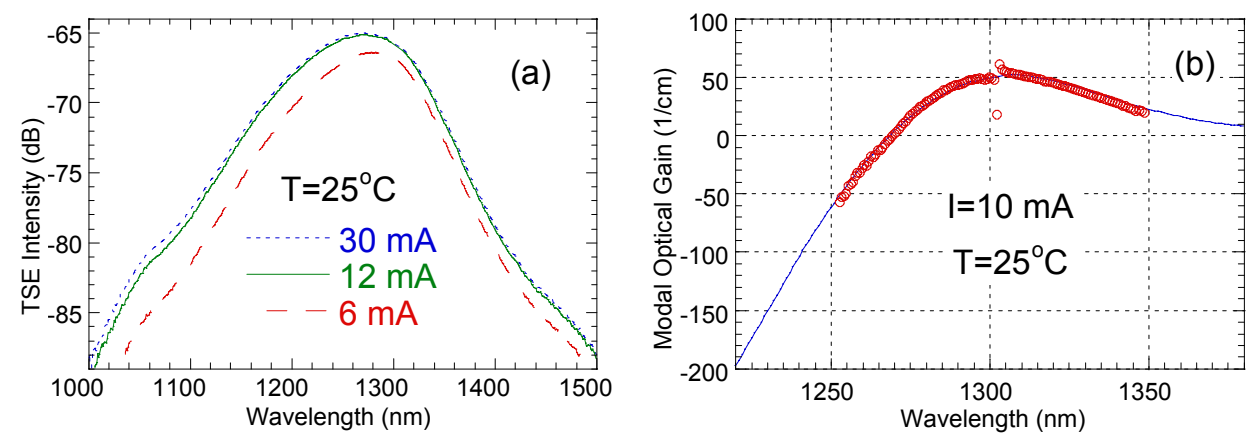

Fig. 2.2. The TSE spectra at $25^{\circ} \mathrm{C}$ for different currents (a) and modal optical gain spectrum (b) extracted from TSE spectra using the Henry technique (solid line). The gain spectrum extracted from the ASE spectrum taken at the same conditions is also shown (circles).

The ASE spectrum of a DFB laser is distorted in the vicinity of the DFB wavelength, and so is the gain spectrum determined from it. The modal optical gain curves extracted from the TSE and the ASE lie very close to each other outside the vicinity of DFB wavelength. This confirms the validity of both techniques. Some discrepancy may arise partly from the fact that the total loss is a weak function of both energy and carrier concentration but was considered constant.

The significant advantage of the methods allowing for extraction of the modal optical gain from the ASE is that they give the absolute value of the gain, and, together with the transparency measurements, the total optical loss. These measurements are fairly easy and fast. The disadvantage is that the modal optical gain is measured in fairly small intervals of both wavelength and gain. When the modal optical gain becomes too low (typically below $150 \mathrm{~cm}^{-1}$ ), the ripple contrast in the ASE spectrum is usually too small to be measured and analyzed accurately. Another disadvantage of these measurements is that the experimental samples must have a Fabry-Perot resonator.

The Henry technique uses the TSE spectra, which is not affected by the value or the spectral dependence of the mirror loss or grating. Therefore it can provide the information about the optical gain in cases when techniques using the ASE are not suitable. Another advantage is the much wider spectral range of measurements. This allows for applications such as analysis of the change of the real part of the refractive index $[4,21]$, that has many important applications. Change of the real part of the refractive index is proportional to the change of the effective refractive index, which defines the position of the lasing wavelength of the laser. For these studies only the 
relative value of change of the optical gain is important, so the Henry technique can be used directly.

\subsection{Determination of the gain in broad-area lasers}

The optical gain spectra can be determined using optical gain spectroscopy techniques $[22,23,24]$. This method utilizes the amplification of the spontaneous emission in an optically pumped active region of variable length. The data are fitted to a calculated linewidth produced by a model of the energy bands in the material to determine the gain as a function of carrier density. These techniques are useful before the device has been processed and provide information on the intrinsic properties of the material.

In [18] the gain spectra were determined using the fundamental relationships between the rates of spontaneous and stimulated emission and optical absorption. Gain was determined for bulk GaAlAs laser structure by measurements of the spontaneous emission observed transverse to the laser cavity for various levels of injected current, supplemented by measurements of the differential quantum efficiency and laser line energy at threshold. The gain value at maximum gain spectra was defined based on the fact that, at the lasing wavelength, the gain at threshold is a maximum and equal to the losses in the cavity. This method required knowledge of the internal efficiency value. In [25] this method was applied to a broad-area InGaAs/InGaAsP quantum-well laser.

The more direct method of the determination of the gain in broad area lasers was described by D. J. Bossert and D. Gallant [26]. Based upon far-field spatial filtering technique, the modal gain change with injection current was determined from belowthreshold ASE spectra. Single lateral mode was separated by spatial far field pattern, and then Hakki-Paoli approach was used to determine value of the modal gain from the fringe contrast in InGaAs/GaAs [27] and midinfrared laser [28].

\section{Measurement of the optical loss}

Internal optical loss is a fundamental characteristic of a semiconductor laser. The value of internal loss affects threshold current and external slope efficiency, and is, therefore, an important parameter. The optical loss has a number of different contributions; most important among them are the free carrier (intraband) absorption [29] and scattering loss on the waveguide nonuniformities.

An early (and still widely used) technique for measurement of internal optical loss was proposed by J. R. Biard et al [30] and extended by H. Casey et al [31]. It requires a set of lasers, varying in length but otherwise equivalent, in order to estimate the average value of loss as well as the internal quantum efficiency. This method does not take into account the systematic variation of the threshold condition due to variation in length and random variation between lasers. Also injection efficiency might not be a constant value across the set of different samples due to incomplete Fermi level pinning resulting from the carrier leakage [32]. Therefore this method could be used for rough evaluations of optical loss, but for the detailed studies more accurate procedure is desirable.

Recently several new techniques have been proposed for optical loss measurements $[33,34,35$, and 11]. These are all based on the Eqs. (2.3) and (2.4) relating the modal optical gain, the material optical gain and the total loss in the laser. From Eq. (2.3) it is clear that the modal optical gain is equal to the total loss (with opposite sign) if the material optical gain is zero. This condition holds to good approximation for the energies 
well below the bandgap energy and holds rigorously at the transparency energy, i.e., at the transition point between absorption and gain [34]. Thus, finding the modal gain at these points provides the value of the total loss, which, in turn, provides internal loss if the mirror loss is known.

A technique based on below-bandgap measurements $[34,35]$ is made difficult by the low intensity of ASE in this region, which limits the accuracy of the gain measurements. For the gain spectra shown in Fig. $2.1 \mathrm{~b}$ the accuracy is no better than $2-5 \mathrm{~cm}^{-1}$. Below bandgap loss measurements are thus adequate for rough estimates of loss, but become unacceptable for more demanding purposes.

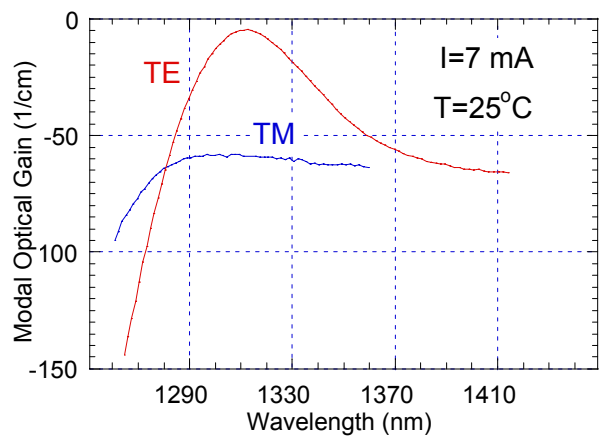

Fig. 3.1. Modal Optical Gain extracted from ASE spectra of MQW laser in TE and TM polarizations.

Other methods reduce the problem of measuring internal loss to the task of determining the material transparency condition. E. Avrutin et al [34] proposed to determine transparency at a given current is to find the intersection of the gain curves in $\mathrm{TE}$ and TM polarizations under the assumption that the optical gain in different polarizations is equal when the material gain is zero. Fig. 3.1 shows the curves of TEand TM-polarized-gain measured for a typical MQW laser. There is one intersection point at high energy (short wavelength) as well as an indication that the two curves will converge at below-bandgap energies as the material optical gain tends toward zero. The possible source of an error of this method is the assumption that the total optical loss is equal in the TE and TM polarizations. This may lead to an error, in this case of about $2 \mathrm{~cm}^{-1}$.

P. Andrekson et al. [33] demonstrated the use of a modulated external probe laser to find the transparency condition, using the optical detection properties of the laser medium [36]. In this method, an $\mathrm{AC}$ voltage induced on the terminals of the laser under test changes polarity when the DC bias current passes through a value corresponding to transparency at the wavelength of the probe laser.

Equivalently, the wavelength of the probe laser can be swept through transparency at a fixed DC bias current. Fig. 3.2a shows a typical plot of the dependence of the induced voltage on the probe wavelength. Also plotted (dotted line) is the ASE spectrum of the laser (this is the same laser sample as in Fig. 3.1). Corresponding features appear in both spectra due to the Fabry-Perot cavity resonances of the laser under test, which enhance the detection properties of the laser at the wavelengths of Fabry-Perot resonances. 

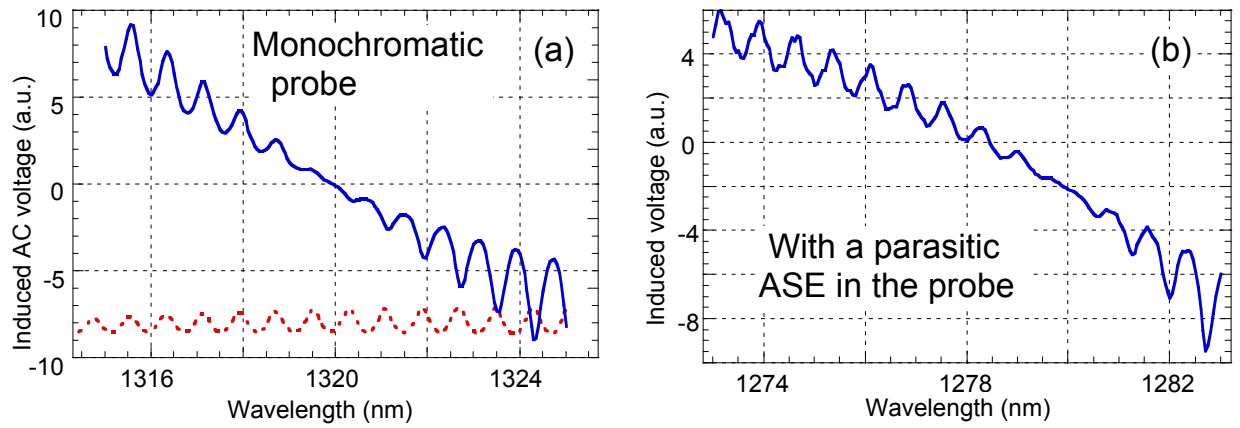

Fig. 3.2. Dependence of the induced AC voltage on the laser terminals on the wavelength of the external probe light in the absence (a) and presence (b) of the parasitic signal. Corresponding part of the ASE spectrum is also shown (dashed line).

It was demonstrated that the external probe measurements are very sensitive to the spectral purity of the probe signal [11]. Some commercially available tunable external cavity lasers (ECL) do not have sufficient signal-to-noise ratio (SNR), which is usually degraded by the presence of the parasitic ASE in the ECL signal. This may result in an error in determination of the transparency wavelength and, therefore, optical loss. To avoid this parasitic effect it was suggested to use the fact that the amplitude of the resonances in the induced voltage spectrum depends on the value of the material optical gain and equals zero at the material transparency. In the absence of any parasitic ASE component in the probe signal, as in Fig. 3.2a, the resonances in the voltage signal vanish at the same probe wavelength at which the induced $\mathrm{AC}$ voltage changes polarity. The effect of low spectral SNR is illustrated in Fig. 3.2b. In this case, the ECL, operating at the edge of its range, provided insufficient suppression of its own ASE. Fig. 3.2b shows an offset of $\sim 2 \mathrm{~nm}$ between the probe wavelengths for which the induced AC voltage equals zero and the wavelengths at which voltage resonances vanish. This difference translates to about $7 \mathrm{~cm}^{-1}$ difference on a gain/loss scale.

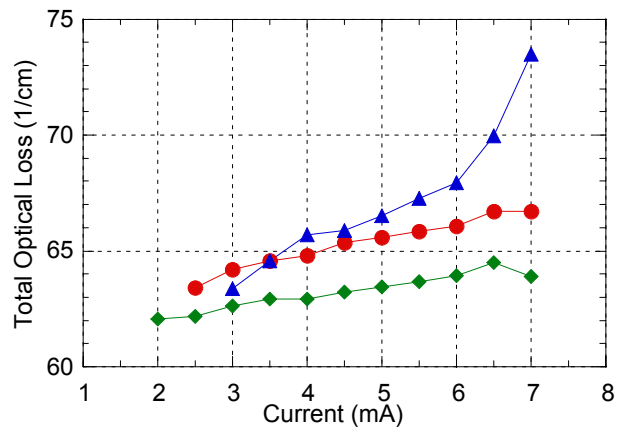

Fig. 3.3. The total loss determined with different methods: using the "zero-voltage" technique (triangles), using the "zero-contrast" rather than "zero-voltage" criterion with the same probe source (circles), and using the TETM crossing method (diamonds).

Fig. 3.3 shows the total loss data measured in three ways: using the "zero-voltage" technique (triangles), using a "zero-contrast" rather than "zero-voltage" criterion with the 
same probe source (circles), and using the TE-TM crossing method (diamonds) discussed above. The effect of the inadequate and varying spectral purity of the probe source gives a serious error in magnitude of total loss, and its dependence on bias current as seen at the lowest and highest currents for the "zero-voltage" set of data. The TE-TM crossing technique gives somewhat better results, underestimating the value of the loss due to the reason explained above, but allowing data to be obtained over a slightly wider range. Smoothly varying and reproducible loss data were observed when using the "zerocontrast" method. The estimated accuracy of this measurement is about $0.5-1 \mathrm{~cm}^{-1}$.

This method made it possible to carry out the study of the loss dependence on temperature and carrier concentration [13]. It was shown that optical loss is a weak function of both temperature and carrier concentration and does not play an important role in determining the dependence of the laser output characteristics (such as threshold current and output efficiency) on temperature. This method is also useful in a process of optimizing the laser structure, when understanding the dependence of the loss on design parameters is important. Another advantage of this technique is that it also provides a very accurate way of determining the transparency energy.

\section{Measurement of the carrier leakage in semiconductor heterolasers}

Performance of semiconductor lasers depends on heterostructure injection efficiency or, in other words, on the fraction of injected carriers consumed by the active region. One of the mechanisms allowing carriers to recombine outside of the active layer is related to thermoionic emission of electrons from the active layer to the p-cladding. This phenomenon is usually referred to as heterobarrier leakage. Another mechanism of carrier escaping from active region prior their recombination is lateral transport of carriers through the blocking structure (in the case of CMBH type devices) or the effect of carrier spreading for broad area lasers. Carrier leakage can affect the laser slope efficiency resulting in its reduction with increase in current and temperature and can contribute to the temperature dependence of the threshold current. The carrier overflow into the barriers and the separate confinement heterostructure has received a great deal of attention due to the influence of carrier concentration in SCH layer on the static and dynamic properties of MQW lasers [37, 38, 39]. The most frequent objects for studies of carrier leakage are InGaAsP/InP heterostructures due to the importance of their high temperature applications and due to the low value of the conduction band discontinuity between the InP cladding layer and the conduction band of the quaternary InGaAsP materials of the waveguide. The effect of heterobarrier leakage on the performance of blue-green and midinfrared lasers was also studied [40, 41, 28]. In this section we will discuss the experimental approaches to studying carrier leakage phenomena in semiconductor lasers focusing our attention on studies of heterobarrier leakage.

\subsection{Optical technique of studying the carrier leakage}

The obvious method of studying the efficiency of carrier leakage under different conditions is to register the light resulting from recombination of carriers outside the active region $[42,43,44,45,46]$. Electro-luminescence image of InGaAsP/InP CMBH laser at $100 \mathrm{~mA}$ forward bias, recorded by E. J. Flynn and D. A. Ackerman is shown in Fig. 4.1a for a leaky laser and in Fig. 4.1b for a device with low leakage. The detected radiation is InP band to band recombination $\lambda=0.97 \mu \mathrm{m}$. Low and high acceptor levels in their p-InP cladding layers characterize these high and low leakage devices respectively. 
One of the earliest experiments in which the intensity of thermionic flow of carriers out of the active region in InGaAsP/InP heterostructure was measured under different conditions is described by E. J. Flynn [46]. In $1.3 \mu \mathrm{m}$ LED structure electrons leaking from the active layer into the p-InP cladding were confined in a subsidiary quaternary layer (leak detection layer). This quaternary layer has a different composition from the active region and the recombination emission caused by carrier leakage $(\lambda=1.05 \mu \mathrm{m})$ was optically detected. Experimental results [42, 43, 44, 45, 46] showed that significant electron leakage can occur in InGaAsP/InP double heterostructures constituting one of the mechanisms of sublinearity of the light-current characteristics and also of the temperature dependence of the threshold current in laser diodes.
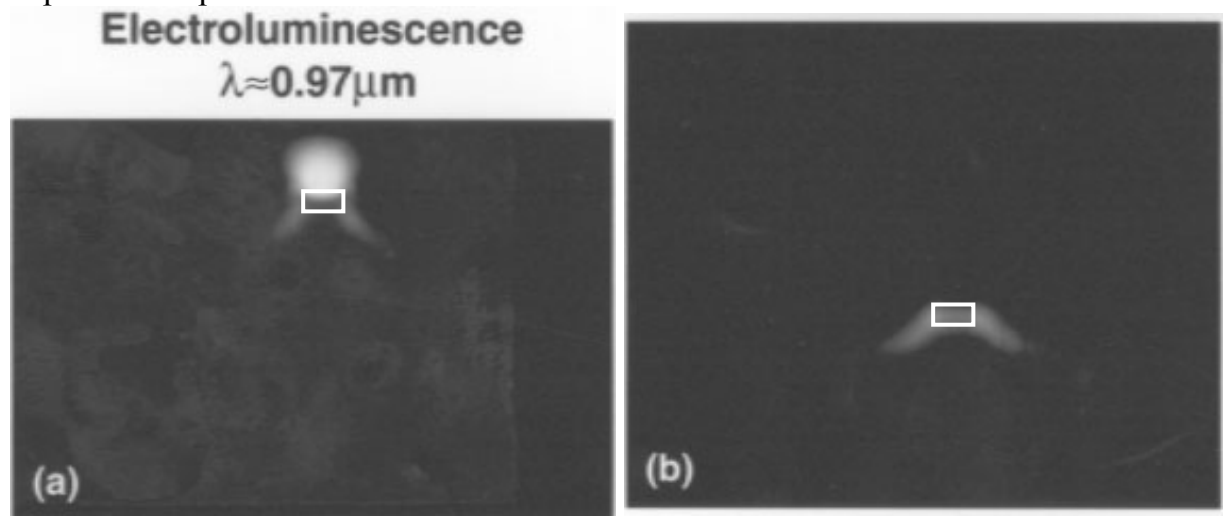

Fig. 4.1. Electrolumininescence images at $100 \mathrm{~mA}$ forward bias: (a) leaky laser and (b) very low leakage laser. Boxes approximately define the position of the active layer. The detected radiation is InP recombination light [46] (Courtesy of E. J. Flynn).

The method of spectrally resolved luminescence was used to study heterobarrier carrier leakage in $\mathrm{CMBH}$ lattice matched and compressively strained $1.3 \mu \mathrm{m}$ InGaAsP/InP lasers with an active layer of the order of $1 \mu \mathrm{m}$ in width [44]. The waveguide forming a step graded SCH consisted of two quaternary materials with slightly a different composition corresponding to the band gaps of $1.1 \mu \mathrm{m}$ and $1.15 \mu \mathrm{m}$ respectively. The intensity of the corresponding emission lines allows conclusions about carrier concentration in different parts of the waveguide. Spontaneous emission spectra were measured in the range of injection currents $1-100 \mathrm{~mA}$ and heat-sink temperatures 20 $80^{\circ} \mathrm{C}$ through the window opening in the contact metallization. It was shown that barrier emission intensity continues to increase even above threshold. Measurements of the temperature dependence of the slope efficiency and the intensity of the emission caused by electron leakage at different temperatures and different pumping currents above the laser threshold were performed [44]. The results indicate that the increase of the carrier leakage in MQW $1.3 \mu \mathrm{m} \mathrm{InGaAsP/InP}$ lasers correlates quantitatively with decreased high temperature slope efficiency. Laser structures with low carrier overflow into barriers and SCH layer exhibit better high-temperature performance. Experiments [44] show that higher values of compressive strain would have to be applied in order to reduce the carrier overflow into the barriers and into the separate confinement region. 


\subsection{Electrical technique of studying the carrier leakage}

Observation and measurements of the leakage current in $1.3 \mu \mathrm{m}$ InGaAsP/InP lasers with the bulk active region by an electrical technique was carried out for the first time by T. R. Chen [47] employing a laser-bipolar-transient-type device. A schematic representation of the device used in this work is shown in Fig. 4.2.

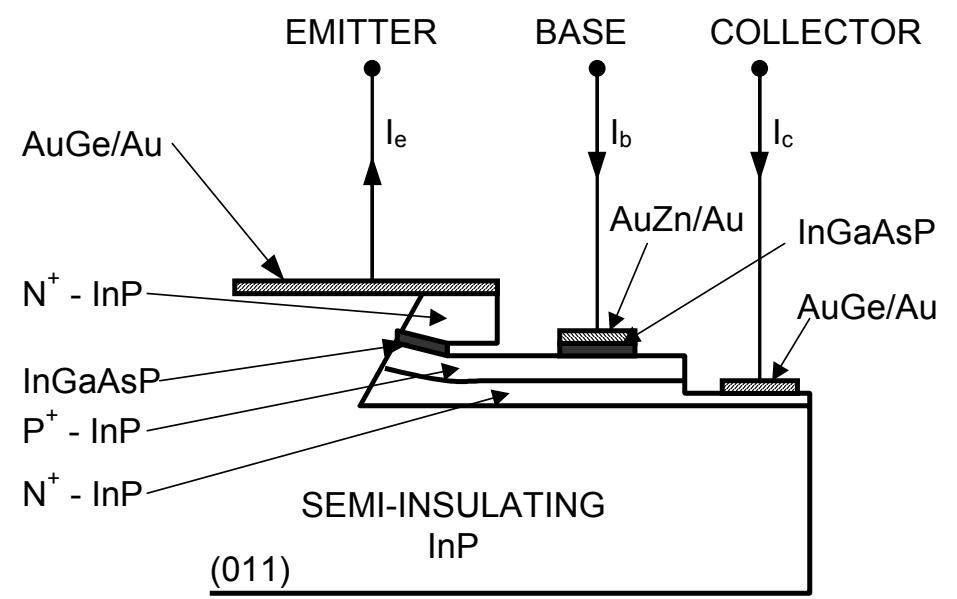

Fig. 4.2. Schematic representation of the laser-bipolar-transistor structure [47].

The emitter base junction functions as in an ordinary laser diode. The collector-base junction is reverse biased, as in a bipolar transistor, to collect the leakage current. The laser part of the transistor was not different from that of a terrace mesa double heterostructure laser. However, an extra $\mathrm{N}^{+}$-InP layer was added in order to collect the heterobarrier electron leakage current. Under forward bias condition, the emitter-base junction acts as a laser diode. The electrons are injected from the InP emitter into the quaternary region. In this structure, the electrons that overcome the heterobarrier A and arrive at the base collector junction B are swept out by the electrical field in the reversebiased junction. A more complex structure to measure the heterobarrier leakage by an electrical method was implemented in [48]. In addition to conventional InGaAsP/InP laser diode layers, the device contains a small area collector, whose layer structure is shown in the inset to Fig. 4.3. 


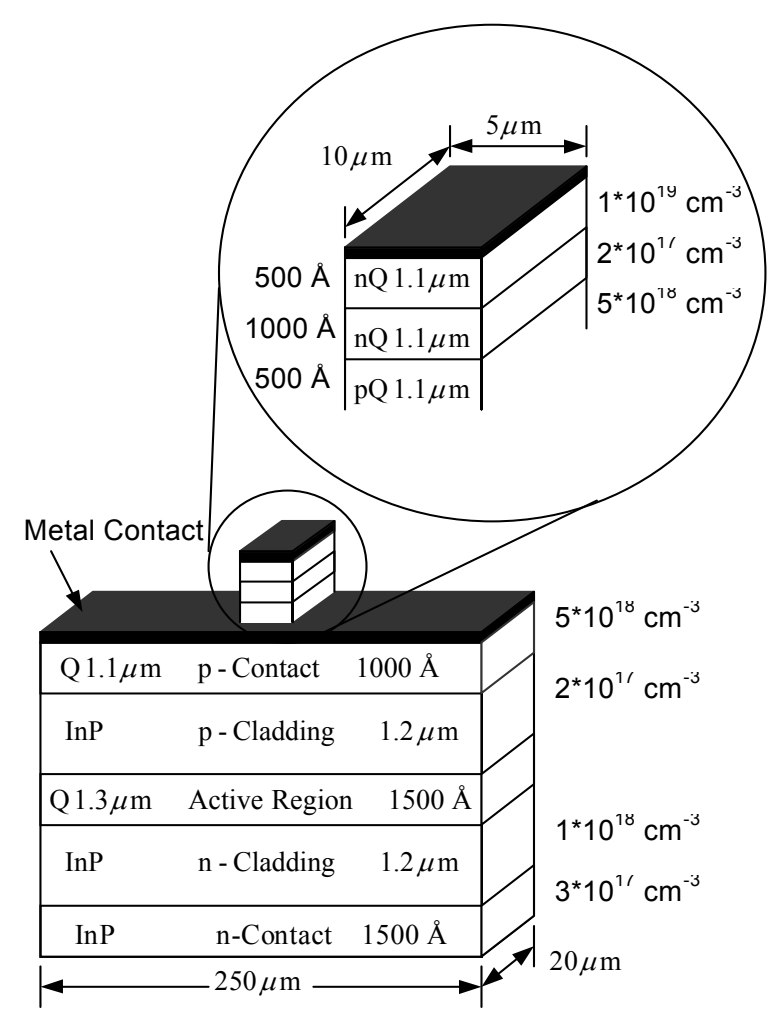

Fig. 4.3. Schematic cross-section of the laser with leakage collector [48].

Fig.4.4 displays the energy band diagram in the cross-section of the device. The diagram corresponds to the operating bias regime and illustrates the idea of the experiment.

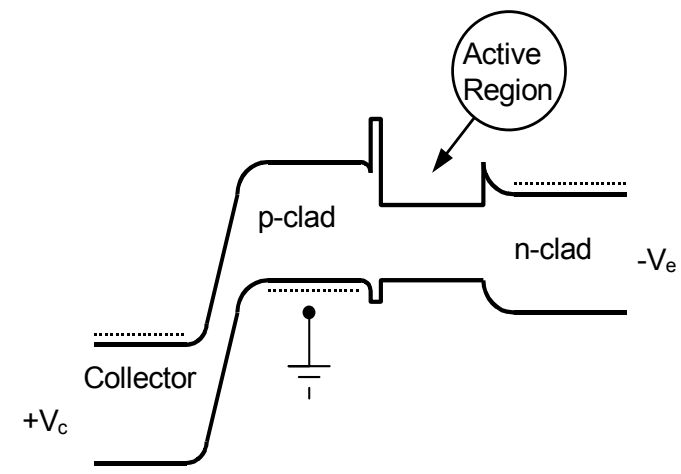

Fig. 4.4. Structure band diagram of the device shown on Fig.4.2. under bias [48].

Since the collector area was only $1 \%$ of the diode area, the presence of the collector does not affect the laser performance. The crucial fabrication step in the processing of the structure in Fig. 4.2 was evaporation of a self-aligned metal contact to the p-quaternary 
layer, which had to provide an ohmic contact to the laser diode without degrading the collector p-n junction.

Typical dependence of the leakage current on injection current for the $1.3 \mu \mathrm{m} \mathrm{MQW}$ InGaAsP/InP broad area lasers with moderately doped p-cladding/SCH interface at different operation temperatures presented in Fig. 4.5 [50]. The use of a three terminal laser structure for systematic studies of the nature of the performance degradation of $1.3 \mu \mathrm{m} \mathrm{MQW} \mathrm{InGaAsP/InP} \mathrm{lasers}[49,50]$ allowed to confirm the theoretical prediction [51], that an increase of the acceptor concentration in the p-cladding layer adjacent to the active region or SCH layer allows to minimize the voltage drop at the p-cladding/SCH interface in these lasers. The reduction of this voltage drop allows to avoid a substantial reduction of the barrier for thermionic emission of electrons from the active region.

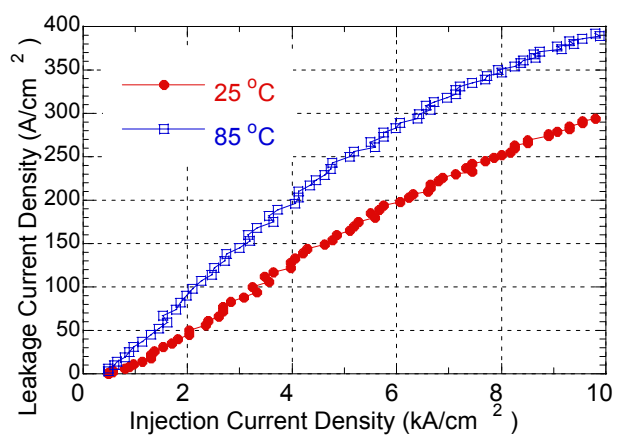

Fig. 4.5. Measured heterobarrier leakage current versus injection current for $1.3 \mu \mathrm{m} \mathrm{MQW} \mathrm{InGaAsP/InP} \mathrm{lasers}$ with moderately doped p-cladding/SCH interface [50].

\section{Electrical and optical measurements of RF modulation response below threshold}

Most of the experimental methods aimed at determining the carrier concentration are based on a model using the balance equations of carriers and photons in the laser. These equations, also known as "Rate Equations", can be derived from the Maxwell equations (see Statz and deMars [52] and Kleinman [53]), and in a simplified single mode case can be written as:

$$
\begin{aligned}
& \frac{d n}{d t}=\eta_{\text {int }} \frac{I}{e V_{a c t}}-R-\frac{c}{N_{e f f}} \frac{\frac{d G}{d n}\left(N-N_{t r}\right)}{1+\in S} S \\
& \frac{d S}{d t}=\frac{c}{N_{e f f}} \Gamma \frac{\frac{d G}{d n}\left(N-N_{t r}\right)}{1+\in S} S-\frac{S}{\tau_{p}}-\beta \Gamma R_{s p}
\end{aligned}
$$

where $n$ and $S$ are the carrier and photon concentrations respectively, $I$ is the pumping current, $e$ is the electron charge, $R$ is the total recombination rate (without stimulated emission), $R_{s p}$ the spontaneous emission rate, $c$ the light velocity in vacuum, $N_{\text {eff }}$ the effective refractive index, $G$ the material optical gain in the active layer, $\Gamma$ the optical confinement factor, and $\tau_{p}$ is the photon lifetime. Internal efficiency $\eta_{\text {nit }}$ accounts for an 
imperfect current injection efficiency into the active layer. The term $(1+\in S)$ describes the gain saturation with power, $\in$ being the phenomenological gain compression parameter. It was first introduced by T. Koch and R. Linke [54] in order to characterize the spectral hole burning. In general this parameter may be used to describe the reduction of the optical gain above threshold due to any other process, such as spatial hole burning, carrier heating etc. In the Eq. (5.1) $\in$ is a composite value, which allows characterizing the reduction of the optical gain due to all the above effects in linear approximation. Since the rate equations in the form of Eq. (5.1) treat the laser as a medium with single spectral and spatial mode, only the value of the gain at the lasing wavelength is important. Therefore the wavelength-independent phenomenological parameter $\in$ can be used. It should be noticed, however, that the use of this parameter is not valid when considering the effects that involve the gain spectra, since different effects distort the gain spectra differently and nonuniformly.

The first equation in the set (5.1) describes the balance of carrier plasma. The carriers are injected by the injection current (first term), and then recombine spontaneously or nonradiatively (second term), as well as through stimulated emission of radiation (last term). The second equation describes the balance of photons inside the laser cavity. In this set of equations two important processes are ignored: carrier transport through the $\mathrm{SCH}$ and MQW layers [55] and carrier capture into quantum wells (in case of QW lasers) $[56,57]$.

The stimulated emission term in the first equation in (5.1) can be neglected below threshold. Then, expressing the recombination in terms of the carrier concentration and carrier lifetime $\tau_{s}$ :

$$
\frac{d n}{d t}=\eta_{\text {int }} \frac{I}{e V_{a c t}}-\frac{n}{\tau_{s}}
$$

For small-signal analysis R. Olshansky et al suggested using a small-signal pulse [58] or sinusoidal [59] excitation. Equation (5.2) can be modified for this case:

$$
\frac{d \delta n}{d t}=\eta_{\text {int }} \frac{\delta I}{e V_{\text {act }}}-\frac{\delta n}{\tau_{d}}
$$

The total number of carriers can be found by integrating the differential carrier lifetime over the bias current [60]:

$$
n(I)=\eta_{\text {int }} \cdot \int_{0}^{I} \tau_{d}\left(I^{\prime}\right) d I^{\prime}
$$

Various techniques, based on the formulae (5.2-5.4) were used to measure the carrier concentration and the recombination rates in semiconductor lasers. A number of authors studied these parameters through carrier lifetime measurements [58, 59, 60, 61, 62]. These measurements use different approaches but all give as a result the value of the carrier lifetime. This value and its dependence on the current characterize various recombination mechanisms in the active layer of semiconductor laser. The major mechanism is the spontaneous radiative recombination - a standard bimolecular recombination. Non-radiative processes such as trap or interface and Auger recombination have also been addressed. 


\subsection{Determination of the differential carrier lifetime from the device impedance}

The equivalent circuit of a semiconductor laser diode in the small-signal modulation regime below threshold is shown in Fig. 5.1. It can be derived from the rate equations discussed above [63,64]. The active layer can be represented as a $R C$ circuit with a characteristic time equal to the differential carrier lifetime.

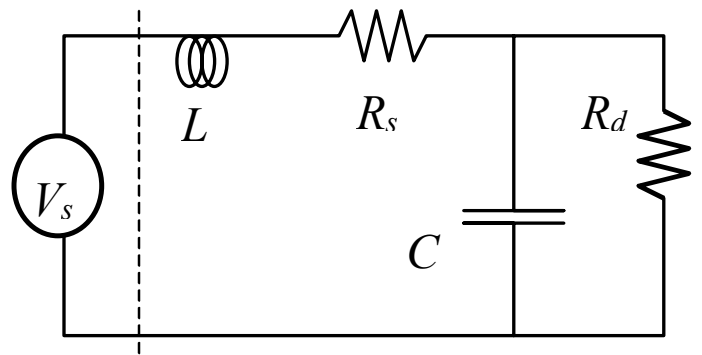

Fig. 5.1. Simple equivalent circuit of laser diode below threshold.

Taking into account a series resistance, $R_{s}$, introduced by the contacts and cladding layers, and a bonding wire inductance $L$, we have a circuit with the total impedance of

$$
Z(\omega)=j \omega L+R_{s}+\frac{R_{d}}{1+j \omega \tau_{d}}
$$

where $\tau_{d}=R_{d} C$, and $R_{d}$ is the static differential resistance of the $p$ - $n$ junction. This model does not take into account leakage paths and a blocking structure capacitance. It also considers that transport and capture-escape times are much faster than the differential carrier lifetime and may then be neglected.

Eq (5.5) has two important consequences. First, the laser impedance below threshold is frequency dependent and therefore the amplitude of the current modulation is not constant over a broad frequency range if a standard signal generator (equivalent to a voltage source) is used in the small-signal response experiments $[12,65]$. Secondly, the differential carrier lifetime can be extracted directly from electrical measurements of the laser impedance [66]. The laser impedance as function of frequency can be measured using a network analyzer. The real and imaginary parts of the laser impedance are described by:

$$
\begin{aligned}
& \operatorname{Re}(Z(\omega))=R_{s}+\frac{R_{d}}{1+\left(\omega \tau_{d}\right)^{2}} \\
& \operatorname{Im}(Z(\omega))=\omega L-\frac{\omega \tau_{d} R_{d}}{1+\left(\omega \tau_{d}\right)^{2}}
\end{aligned}
$$

Plotted in Fig. 5.2 are measured real (a) and imaginary (b) parts of the impedance of a laser with the bulk active layer at room temperature and current of $1 \mathrm{~mA}$. The solid lines are fits to the Eqs. (5.6) and (5.7) with the parameters shown. Fitting the real and imaginary parts of the laser impedance gives very close values for all model parameters.

The measured laser impedance shows a frequency-independent series component, $R_{s}$, of approximately $5 \Omega$ and a frequency- and bias-dependent component, $R_{d}$, associated with the differential impedance of a diode $p-n$ junction $[63,64,67]$. The magnitude of both components can also be measured as a function of current in the $\mathrm{CW}$ regime: $R_{s}$ by 
extrapolating the above-threshold value of $d V / d I$ to threshold and $R_{d}$ by measurement of the sub-threshold value of $d V / d I(I)-R_{s}[68]$. These values were found to be very close to those extracted from the RF measurements [12].
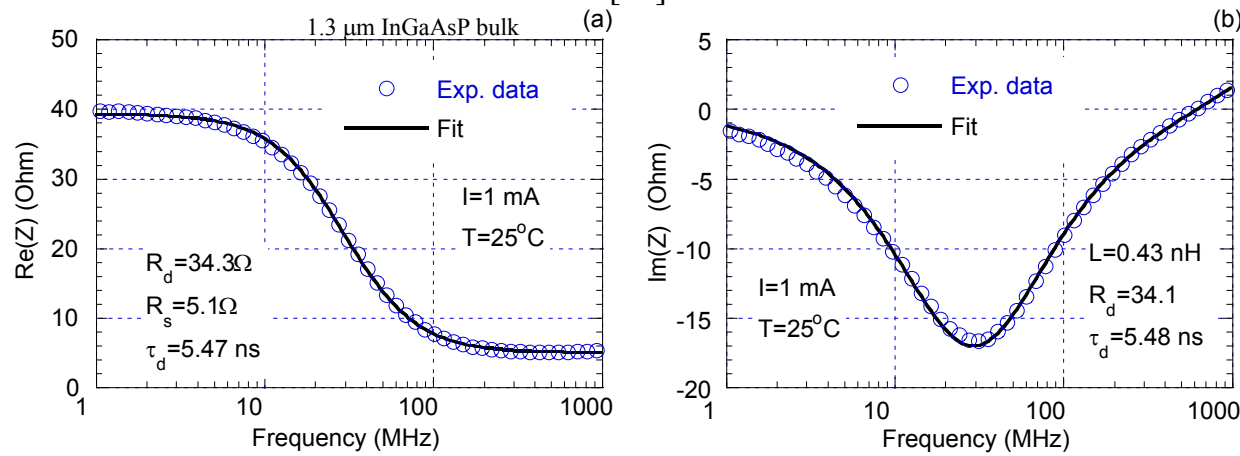

Fig. 5.2. Measured real (a) and imaginary (b) parts of the laser diode impedance (circles) and fit to Eq. (5.6-5.7) (solid lines).

This measurement technique is based on a simple purely electrical measurement. It is useful when optical detection is difficult, (e.g. at low currents and in the wavelength ranges where no fast detectors are available). On the other hand, this method is not very accurate at high currents, where the value of the differential resistance, $R_{d}$ becomes diminishingly small. This model in general is correct only as long as the transport effects including the capture-escape process $[55,56,57,69]$ can be neglected. In the case of highly doped MQW lasers this model is not accurately correct and more complicated model is required $[56,57]$.

One more important limitation of this technique is that the laser diode itself might have parasitic capacitance formed by the contact pads, blocking layers etc. This capacitance is effectively connected in parallel with the laser $p$ - $n$ junction capacitance, $C$, in the equivalent circuit of Fig. 5.1. It is usually thought to be small compared with the junction capacitance, but the latter one decreases as the current through the laser decreases, and at some current the junction capacitance may become comparable to the parasitic one. Then equivalent circuit of Fig. 5.1 is no longer valid.

\subsection{Determination of the differential carrier lifetime from the optical response measurements}

The original technique for determination of differential carrier lifetime proposed to use a small-signal current step excitation [58]. The optical response curve was fitted to an exponential form based on Eq. (5.3). This technique has a disadvantage of high noise if the excitation signal is small. A solution is to use a frequency domain analysis of Eq. (5.3), which allows the use of a RF spectrum analyzer, giving superior signal-to-noise ratio for the same levels of excitation. Equation (5.3) has a solution in the frequency domain:

$$
\delta n(\omega)=\frac{\delta I(\omega)}{e V_{a c t}} \frac{\tau_{d}}{1+j \omega \tau_{d}}
$$

where $\omega=2 \pi f$ is the cyclic modulation frequency, and $j$ is the complex unit. Under 
small signal modulation, the deviation of the photon concentration $\delta S$ is proportional to the deviation of the carrier concentration $\delta n$.

Originally, many authors used the Eq. (5.8) considering that the current amplitude is frequency independent. However, most of the commercially available signal generators are power sources, which in the experiment are loaded on the laser diode. This connection has an equivalent circuit of a voltage source loaded on an $r=50 \Omega$ resistor plus a laser impedance $Z(\omega)$. The frequency dependence of the impedance results in the frequency dependence of the amplitude of current modulation, therefore it should be taken into account in order to extract a correct value of the differential lifetime [65, 12]. Equation (5.8) can be modified to yield:

$$
\delta n(\omega)=\frac{1}{e V_{a c t}} \cdot \frac{\delta V(\omega)}{r+Z(\omega)} \cdot \frac{\tau_{d}}{1+j \omega \tau_{d}}
$$

The voltage modulation amplitude $d V(\omega)$ can be considered to be constant over the frequency range. Therefore, knowledge of $Z(\omega)$ can be used to correct the measured optical modulation, in order to use the single-pole fitting procedure proposed in [59]. Using the equation (5.9) we can write:

$$
F(\omega)=|\delta S(\omega)| \cdot|r+Z(\omega)| \propto \frac{1}{\sqrt{1+\left(\omega \tau_{d}\right)^{2}}}
$$

where the corrected response function, $F(\omega)$, can be used to extract the differential lifetime. The measured optical response curve (circles in Fig. 5.3) was corrected using Eq. (5.10) (squares in Fig. 5.3), and then fit to a single pole roll-off from which the differential carrier lifetime was extracted.

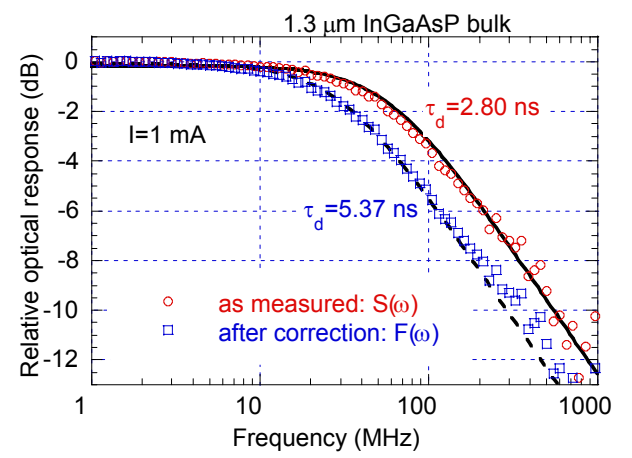

Fig. 5.3. Optical response curves: as measured (circles), and corrected using Eq. (5.10) (squares). Lines are single-pole fits.

The results of the measurements of the differential carrier lifetime using all three of the above techniques are plotted in Fig. 5.4. When the differential carrier lifetime is measured using the uncorrected optical response technique (rhombs), it shows noticeable saturation and decrease at low injection currents. Other authors [59] have observed this behavior. Additional recombination mechanisms were introduced to account for this effect. Comparison of the differential carrier lifetimes obtained using the impedance analysis technique (triangles), and the impedance-corrected optical technique (circles) to the data obtained using uncorrected optical response illustrates the large effect of the rapidly varying laser impedance, especially at low bias current. The corrected differential 
lifetimes give very close values within the entire current range and no longer show the saturation and decrease with decreasing current. There is no need to invoke an extra mechanism of current consumption as described in reference [59]. It can be concluded, that the apparent saturation in lifetimes is due to the finite output impedance of the signal source (together with the high differential impedance of the laser at low currents).

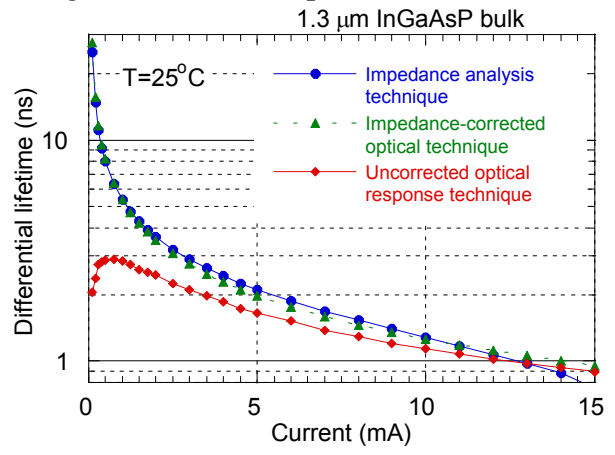

Fig. 5.4. Dependence of differential carrier lifetime (determined using different techniques) on current.

Finally, the simplified model of the laser impedance can be used for correction of the differential lifetime data obtained from the optical response measurements using the values of $R_{d}$ and $R_{s}$ obtained from either static or dynamic measurements [65]. Substituting Eq. (5.5) into Eq. (5.8) gives a corrected differential carrier lifetime:

$$
\tau_{d, \text { corr }}=\tau_{d, \text { opt }} \cdot\left(1+\frac{R_{d}}{R_{s}+r}\right)
$$

in which the corrected lifetime is larger than the uncorrected, optically measured lifetime by the factor in parentheses $(r=50 \Omega$ is the signal source output impedance). For example, this correction factor equals 2 when $R_{d}$ is about $55 \Omega$, which occurs at approximately $0.7 \mathrm{~mA}$. Use of this simple correction gives the same value of the differential lifetime as the technique based on impedance analysis and impedance corrected optical measurement.

\section{Optical measurements of RF modulation response and RIN above threshold}

Small-signal response measurement is a very powerful technique that allows analyzing a number of important device parameters, such as differential gain and gain suppression coefficient. They are also very useful in estimating the device performance in the real operating conditions, such as direct digital current modulation.

The set of Eqs. (5.1) can be solved numerically for large signal analysis. Also, simple small-signal analysis allows for determination of some fundamental characteristics of the laser. In this approximation small sinusoidal current modulation around the static value results in sinusoidal modulation of the carrier concentration and photon density [70, 71]:

$$
\begin{aligned}
& I(t)=I_{0}+I_{1} \exp (j \omega t) \\
& n(t)=n_{0}+n_{1}(\omega) \exp (j \omega t) \\
& S(t)=S_{0}+S_{1}(\omega) \exp (j \omega t)
\end{aligned}
$$


Following [70,71], the solution for optical power response can be written as

$$
\frac{P(\omega)}{I(\omega)}=\frac{h v}{q} \eta H(\omega)
$$

where the two-parameter modulation transfer function $H(\omega)$ is defined as in [2], (p.200):

$$
H(\omega)=\frac{\omega_{R}^{2}}{\omega_{R}^{2}-\omega^{2}+j \omega \gamma}
$$

The two parameters in Eq. (6.3) are the electron-photon resonance frequency $\omega_{R}$ and the damping factor $\gamma$. Expressions for these values are derived in the course of the same analysis:

$$
\omega_{R} \approx \sqrt{\frac{\frac{c}{N_{e f f}} \frac{d G}{d n} S_{0}}{\tau_{p}\left(1+\in S_{0}\right)}}
$$

and

$$
\gamma=\frac{1}{\tau_{s}}+\frac{c}{N_{e f f}} \frac{d G}{d n} \frac{S_{0}}{1+\in S_{0}}+\frac{1}{\tau_{p}} \frac{\in S_{0}}{1+\in S_{0}}
$$

where photon lifetime $\tau_{p}$ is proportional to the total optical loss:

$$
\frac{1}{\tau_{p}}=\frac{c}{N_{e f f}} \alpha_{t o t}
$$

Shown in the Fig. 6.1 are the amplitude (a) and phase (b) of the laser optical modulation response at different DC biases, measured using HP8510 Network Analyzer with HP83440D high-speed p-i-n detector. The response curves show typical resonant behavior described by the Eq. (6.3). Additional drooping of the response curves, seen in the Fig. 6.1a has been attributed to carrier transport through the SCH layers [55] and to the carrier capture and thermoionic emission processes in QW lasers [72].
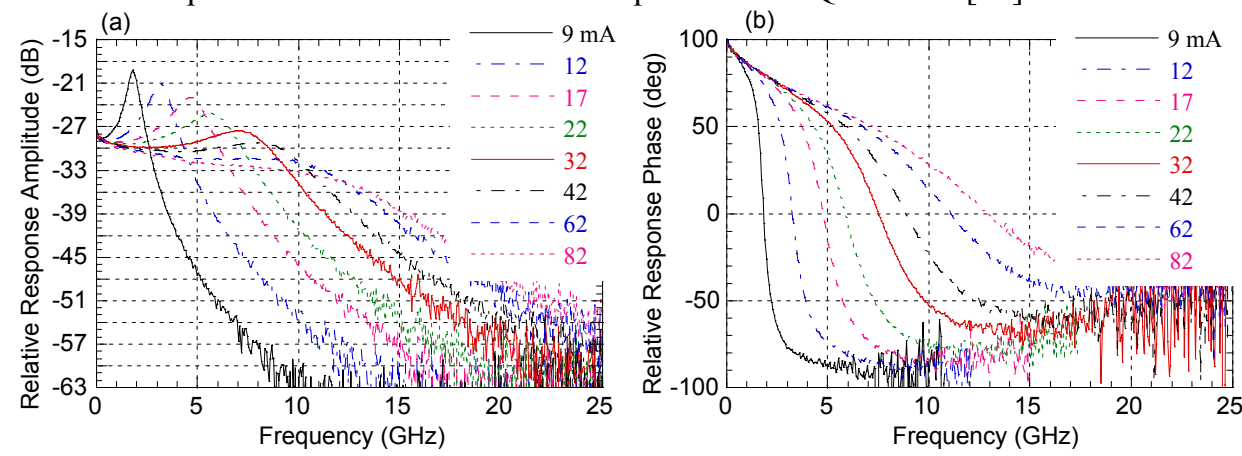

Fig. 6.1. Relative Amplitude (a) and Phase of the laser response to small-signal direct current modulation, measured using p-I-n detector and HP8510 Network Analyzer (at different DC bias currents).

As it can be seen from the Eqs. (6.4-6.5), the resonance frequency and damping factor contain important information on the material parameters: differential gain and the gain 
compression coefficient. If these values were known as functions of the injection current (or optical power), the above parameters could be determined.

The damping factor $\gamma$ increases proportionally to the square of the resonance frequency $\omega_{R}^{2}$. R. Olshanslky et al [73] have defined the proportionality coefficient between the $\gamma$ and $\omega_{R}^{2}$ and as $\mathrm{K}$-factor:

$$
\begin{aligned}
& \gamma=K f_{R}^{2}+\gamma_{0} \\
& K \approx 4 \pi^{2}\left(\tau_{p}+\frac{\epsilon}{\frac{c}{N_{\text {eff }}} \frac{d G}{d n}}\right)
\end{aligned}
$$

The resonant frequency increases with current and so does the small-signal modulation bandwidth (the frequency at which the response amplitude drops by $3 \mathrm{~dB}$ from its DC value), but only up to a certain level. As the DC injection current increases, the response flattens (as it can be seen in the Fig. 6.1a). At some point the damping factor becomes large enough that response amplitude drops by $3 \mathrm{~dB}$ at the frequency below $\omega_{R}$. It was shown [73] that maximum possible bandwidth is approximately:

$$
f_{3 d B \max } \approx \sqrt{2} \frac{2 \pi}{K}
$$

\subsection{Determination of the resonant frequency and damping factor using carrier subtraction technique. Determination of the differential gain and the gain compression coefficient}

The differential gain and the gain saturation parameters can be determined from the dependencies of the resonance frequency and damping factor on the optical power (DC drive current) using the Eqs. (6.4-6.7). These dependencies can both be determined from the small-signal response measurements described in the previous section. However an accurate procedure is not trivial. As it can be seen from the Fig. 6.1a, the amplitude response curves cannot be directly fit to the Eq. (5.14) since there is an additional factor in the transfer function. This additional factor has been associated with the carrier transport; also the response characteristics of the laser can be further distorted by $R-C-L$ parasitic elements in the electrical feed circuit. In general, the response function in the Eq. (5.13) should be written in a more complicated form:

$$
\frac{P(\omega)}{I(\omega)}=\frac{h v}{q} \eta \cdot H\left(\omega, \omega_{R}, \gamma\right) \cdot T(\omega)
$$

where $T(\omega)$ is a composite transfer function described above. This function cannot be written analytically in the general case.

P. Morton has proposed an elegant way to determine the resonant frequency and damping factor from the response measurements. He demonstrated that in the assumption that the transfer function $T(\omega)$ does not depend on the DC bias and the modulation efficiency $I(\omega)$ is constant above threshold, the ratio of two does not depend on any factors other than respective resonant frequencies and damping factors. Since the relative 
response amplitude curves are typically measured in the logarithmic $(\mathrm{dB})$ units, it is convenient to express the difference between the two responses in this way:

$$
\begin{aligned}
& \text { Response }_{1}(f)-\text { Response }_{2}(f)=20 \log _{10}\left(P_{1}(f)\right)-20 \log _{10}\left(P_{2}(f)\right)= \\
& =10 \log _{10}\left(\frac{\left(f_{R 2}{ }^{2}-f^{2}\right)^{2}-\left(f \gamma_{2} / 2 \pi\right)^{2}}{\left(f_{R 1}{ }^{2}-f^{2}\right)^{2}-\left(f \gamma_{1} / 2 \pi\right)^{2}}\right)+\text { const }
\end{aligned}
$$
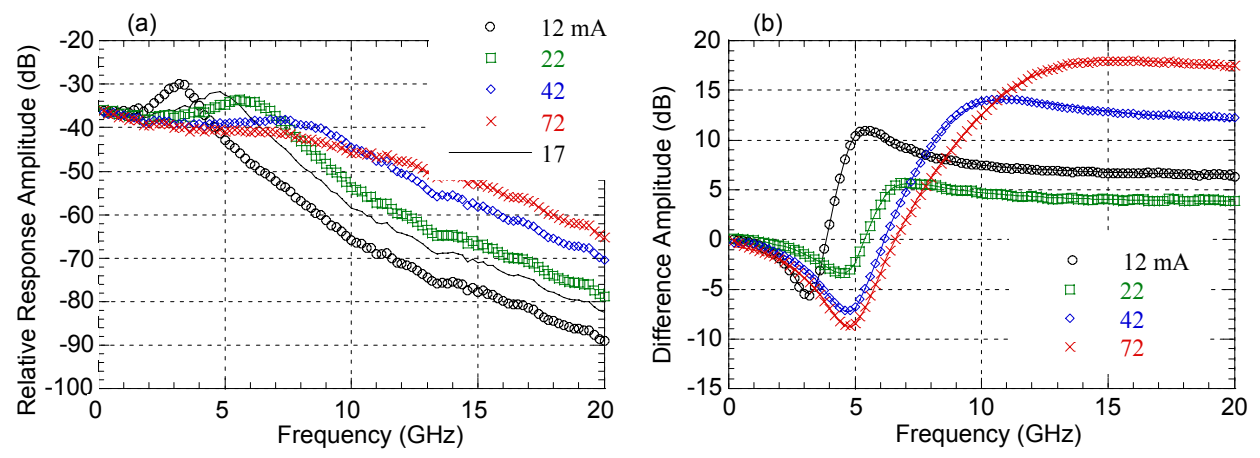

Fig. 6.2. Relative response amplitudes (a) measured using p-i-n detector and RF spectrum analyzer (for the same laser as in Fig. 6.1), and the results of subtraction of the reference curve (solid line in (a)) from the response curves [74]. Solid lines in (b) are fits to the Eq. (6.10).

Shown in the Fig. 6.2a are the relative response amplitudes measured using p-i-n detector and HP71400 RF spectrum analyzer. The data was taken for the same device as in the Fig. 6.1. The signal noise at low amplitude levels and high modulation frequencies is considerably lower than in the case when the measurements were done using the Network Analyzer, because the RF spectrum analyzer has considerably higher dynamic range. This is very useful because the low noise at high frequencies is important for successful fitting of the difference curves to the Eq. (6.10). The response curves in the Fig. 6.2a are not as smooth as in the Fig. 6.1a because of some calibration inaccuracy (in the particular case of the data shown in the Fig. 6.2a this inaccuracy is not very high). But the calibration data is not needed since the calibration correction enters as an additional factor into the function $T(\omega)$. P. Morton had demonstrated [74] that even high calibration inaccuracy is completely eliminated by the subtraction procedure. This feature makes the subtraction technique very valuable for the lasers having high parasitic RC parameters or packaged for DC or low-frequency operation.

The results of the subtraction procedure are presented in the Fig. 6.2b. The differential curves obtained by subtraction of the response amplitudes at $17 \mathrm{~mA}$ from the response amplitudes at each current are shown with the same symbols, as the original curves in the Fig. 6.2a. These differential curves are very smooth and can easily be fitted to the Eq. (6.10) (solid lines). It turns out that even though the curves are fitted to five-parameter expression, the fitting procedure converges very well and gives very accurate data for the resonant frequencies and damping factors.

The results of the curve fitting performed on the differential curves are presented in the Fig. 6.3. Solid curves are linear fits. The same procedure was performed with the differential curves obtained by subtracting the response at different currents to check the 
accuracy of the procedure. The values of the K-factor and differential gain were within $5 \%$ range, which confirms the robustness of the method.
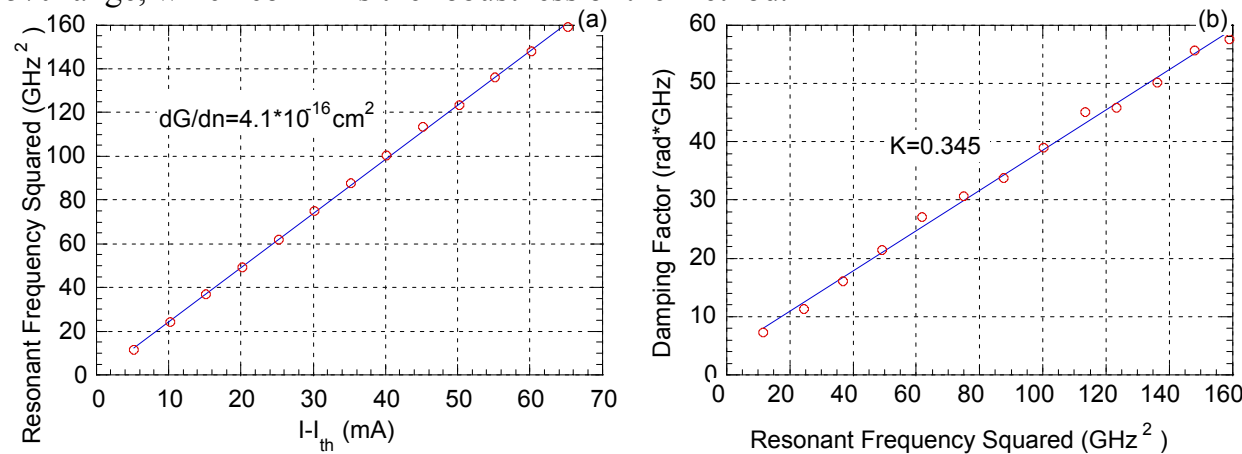

Fig. 6.3. Results of the curve fitting of the differential curves presented in the Fig. 6.2b: (a) Resonant Frequency Squared as a function of the current deviation above threshold, (b) Damping Factor as a function of Resonant Frequency Squared.

Knowledge of the dependence of the resonant frequency on current allows determining the differential gain in the material of the active layer. Using the Eq. (6.4) we can write:

$$
\frac{d G}{d n} \approx \frac{4 \pi^{2} f_{R}^{2}}{\frac{c}{N_{e f f}} S_{0}} \tau_{p}=\frac{4 \pi^{2} e V}{\Gamma \eta_{i} \frac{c}{N_{e f f}}} \frac{f_{R}^{2}}{\left(I-I_{t h}\right)}
$$

where $I_{t h}$ is the threshold current, and we used the relation between the photon density and the output power:

$$
S_{0} \approx \frac{\Gamma}{V} \cdot \frac{P_{\text {out }}}{h v} \frac{1}{\frac{c}{N_{\text {eff }}} \alpha_{\text {mirr }}}=\frac{\Gamma}{V} \cdot \frac{\eta_{i}}{e} \tau_{p}\left(I-I_{\text {th }}\right)
$$

where the mirror loss $\alpha_{\text {mirr }}$ is given by the first term of the Eq. (2.4) for uncoated device.

In the current treatment the effects of the carrier transport and capture into the QW are ignored. More accurate phenomenological treatment yields another factor $\chi$ in the right side of the Eq. (6.11). $\left(\chi=1+\tau_{d} / \tau_{e}\right.$, where $\tau_{d}$ is the carrier capture time including diffusion across SCH and $\tau_{e}$ the thermionic emission lifetime [72]).

Differential gain was estimated from the data presented in the Fig. 6.3a using the Eq. (6.11). Ignoring the transport effects $(\chi=1)$ and assuming the internal efficiency $\eta_{i}=1$, we obtain $d G / d n=4.1 * 10^{-16} \mathrm{~cm}^{2}$.

\subsection{Determination of the resonant frequency and differential gain from the RIN measurements}

Relative intensity noise (RIN) measurements represent the alternative technique used for studies of high-speed dynamics in QW lasers. Like modulation response technique described above this approach allows for determining of $d G / d n$ and $K$-factor through numerical fitting procedure. However, the signal measured is not response to the external 
modulation of the bias current but noise spectra of the laser itself. This noise power is associated with the fluctuation of the concentrations in photon and electron systems caused by spontaneous light emission events. Deviation of the electron and photon densities from equilibrium values leads to their damped oscillations with the frequency of electron-phonon resonance $f_{R}$ and damping factor $\gamma$. Hence, the measurements of the laser noise power spectra at different bias currents provide enough information to determine such important laser parameters as differential gain and $K$-factor.

Relative Intensity Noise (RIN) is defined (see p.221-225 in [2]) as the mean square value of the noise power related to the mean value of the output power squared:

$$
R I N \equiv \frac{\left\langle\delta P(t)^{2}\right\rangle}{P_{0}^{2}}
$$

It is more convenient to define RIN per unit bandwidth because the measurement bandwidth can vary under different experimental conditions. Then:

$$
\frac{R I N}{\Delta f} \equiv \frac{2 S_{\delta P}(\omega)}{P_{0}^{2}}
$$

where $S_{\delta P}(\omega)$ is double sided noise power spectral density (if the spectral density is defined as single-sided, then the factor 2 should be removed). As defined, RIN is measured in $\mathrm{dB} / \mathrm{Hz}$.

Small signal analysis applied to the rate equations similar to the Eq. (5.1) but taking into account internal noise source and transport of carriers leads to the following expression for the spectral dependence of the RIN:

$$
R I N=\frac{4}{\pi} \delta f_{s t} \frac{f^{2}+(\gamma * / 2 \pi)^{2}}{\left(f_{r}^{2}-f^{2}\right)^{2}+f^{2}(\gamma / 2 \pi)^{2}}
$$

where $-\delta f_{s t}$ - Schawlow-Townes linewidth and $\gamma^{*}$ - differs from $\gamma$ in denominator due to carrier transport through the active region of modern MQW laser [72]. RIN spectra for the $1.3 \mu \mathrm{m} \mathrm{InGaAsP/InP} \mathrm{MQW} \mathrm{DFB} \mathrm{laser} \mathrm{at} \mathrm{the} \mathrm{different} \mathrm{bias} \mathrm{condition} \mathrm{is} \mathrm{presented}$ in Fig. 6.4. [75]. The resonance frequency, damping factor and Shawlow-Townes linewidth can be determined from fitting the experimental RIN spectra to the Eq. (6.15).

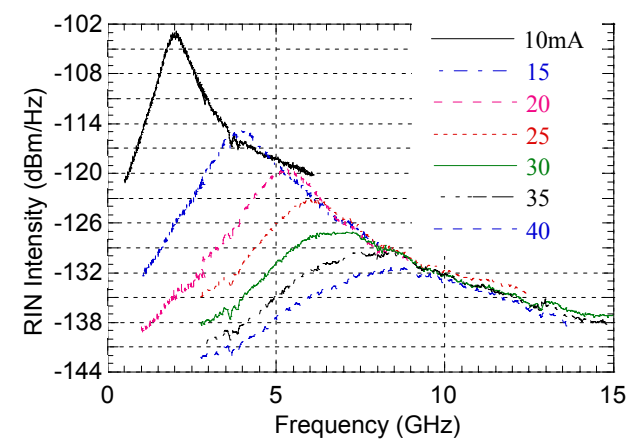

Fig. 6.4. Dependence of the RIN spectra for $1.3 \mu \mathrm{m} \mathrm{CMBH} \mathrm{InGaAsP/In} \mathrm{MQW} \mathrm{DFB} \mathrm{laser} \mathrm{at} 25^{\circ} \mathrm{C}$ on bias current. 
RIN measurement technique was utilized to study the high-speed characteristics of $1.3 \mu \mathrm{m}$ and $1.55 \mu \mathrm{m}$ InGaAsP/InGaAs [76, 77, 78] and $1.55 \mu \mathrm{m}$ InGaAs/InGaAlAs [79] lasers.

As it was mentioned in the previous section, by taking account the carrier transport properties of the structure one can obtain dependence of the resonance frequency on current, which allows to determine the differential gain. Experimental results for the same laser as in Fig. 6.4 are presented in Fig. 6.5. [75]. The values of the differential gain and $K$-factor are also shown [75]).
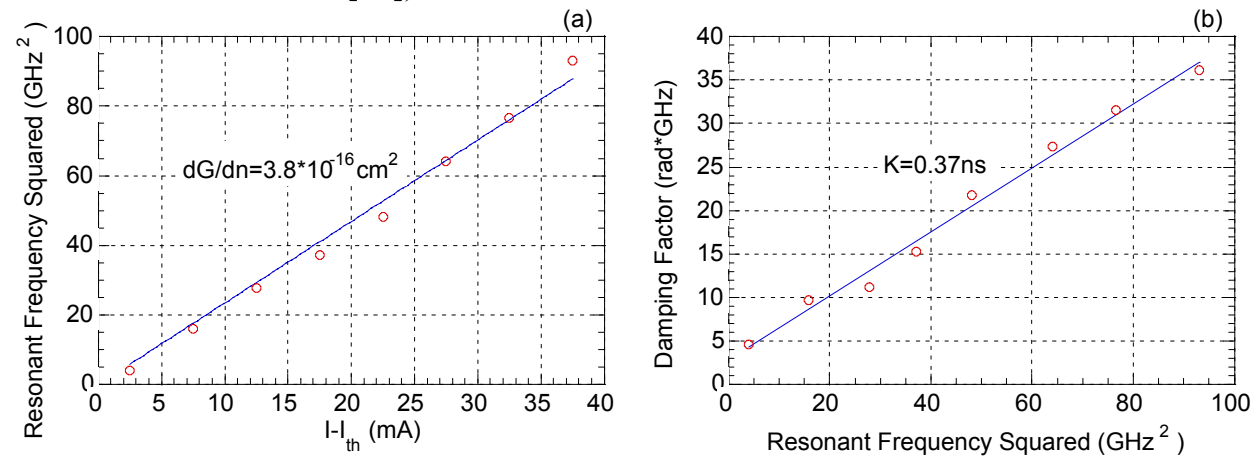

Fig. 6.5. Determination of the differential gain (a) and K-factor (b) from the slopes of the theoretically predicted linear dependencies.

The main advantage of the RIN technique with respect to modulation response measurements is elimination of the problem with high frequency modulation experimental equipment. At the same time, low level of the noise for high quality lasers makes it is necessary to use low noise preamplifiers. Also, there is no low frequency rolloff in the RIN spectra, which is related to the carrier capture and diffusion across SCH time.

\section{Measurements of the linewidth enhancement factor}

Fleming and Mooradian [80] first observed that the static linewidth of the semiconductor laser is much larger than predicted by classical Schawlow-Townes formula. C. Henry has introduced the linewidth enhancement factor [81], which relates small variations of the real and imaginary parts of the refractive index due to a change of the carrier concentration:

$$
\alpha_{T}=\left.\frac{\left.\frac{\partial N^{\prime}}{\partial n}\right|_{T}}{\frac{\partial N^{\prime \prime}}{\partial n}}\right|_{T}=\left.\frac{\partial N^{\prime}}{\partial N^{\prime \prime}}\right|_{T}
$$

C. Henry [81] had shown that spontaneous emission noise in the active layer of the semiconductor laser causes the fundamental broadening of the Lorentzian laser linewidth: 


$$
\Delta v=\frac{c}{N_{\text {eff }}} \frac{R_{s p}}{G} \frac{\alpha_{\text {mirr }} h v}{8 \pi P}\left(1+\alpha_{T}^{2}\right)
$$

where, again, the $P$ is output power, $R_{s p}$ the spontaneous emission rate, and $\alpha_{\text {mirr }}$ the mirror loss. The linewidth enhancement factor is labeled $\alpha_{T}$ here to emphasize the fact that variations are due to a change of carrier concentration at constant carrier temperature.

Linewidth enhancement factor $\alpha_{T}$ is a critical parameter determining both the static linewidth of the semiconductor laser and instantaneous wavelength change of the semiconductor laser under direct modulation, also known as the wavelength chirp. It was shown that for most applications low values of parameter $\alpha_{T}$ are desirable. Determination of the parameter $\alpha_{T}$ is therefore important for understanding and improving the laser design and performance. This can be done using various methods; some related to the gain measurements described in previous sections. C. Henry [4] has proposed to use the Kramers-Krönig relation to determine the $\alpha_{T}$ parameter from the optical gain data obtained from TSE measurements described in the Section 2.

Shortly after C. Henry [81], K. Vahala and A. Yariv developed a theory predicting the same linewidth enhancement factor [82]. C.Harder et al also proposed a method to determine the linewidth enhancement factor $\alpha_{T}$ from the high-frequency modulation experiment [83]. This method involves fair amount of effort since both FM and AM modulation indices have to be measured. FM modulation index measurement at high frequency requires FP interferometer (supercavity detector), which is sometimes inconvenient.

F. Devaux et al [84] have proposed another method for measuring the linewidth enhancement factor using the above threshold modulation response measurements, very similar to those discussed in Section 6.1. They proposed to utilize the FM/AM conversion in a dispersive media such as standard optical fiber. Optical spectrum of the modulated laser (as well as any other source) contains modulation side-bands around central optical frequency. These side-bands have different group velocities in the fiber, and therefore different delays after traveling through some distance of the fiber. This results in sharp drops (nulls) in the response curve at frequencies corresponding to the phase difference of $\pi$ between these side-bands. The exact frequencies of these nulls are determined by both the linewidth enhancement factor and fiber dispersion and can be measured using standard network analyzer. Therefore the linewidth enhancement factor can be determined very accurately. The disadvantage of this method is that it can only be used for characterization of spectrally single-moded sources, otherwise (for example it cannot be used for FP lasers).

\subsection{Measurements of linewidth enhancement factor $\alpha_{T}$ from ASE and TSE spectra}

I.Henning and J.Collins proposed a very simple technique for measuring the linewidth enhancement factor $\alpha_{T}$ based on the ASE measurements [85]. Eq. (7.1) can be used with other equations relating the change of the real and imaginary parts of the refractive index to the values, which can be measured experimentally. Rewriting the Eq. (2.5): 


$$
\delta N^{\prime \prime}=-\frac{1}{\Gamma} \frac{\delta g}{4 \pi} \lambda
$$

and

$$
\Gamma \frac{d N^{\prime}}{N^{\prime}} \approx \frac{d N_{e f f}}{N_{e f f}}=\frac{d \lambda}{\lambda}
$$

where $N_{\text {eff }}$ is the effective group index. Substituting Eqs. (7.3) -(7.4) into Eq. (7.1) we get:

$$
\alpha_{T}=-\left.\frac{4 \pi N_{e f f}}{\lambda^{2}} \frac{\partial \lambda}{\partial g}\right|_{T}
$$

By recording the ASE at different currents below threshold and measuring the change of the gain and the wavelength shift of Fabry-Perot peaks with current one can determine the linewidth enhancement factor $\alpha_{T}$ and its dependence on the wavelength and current. Shown in the Fig. 7.1a is a set of $\alpha_{T}$ factor curves measured using the above procedure.
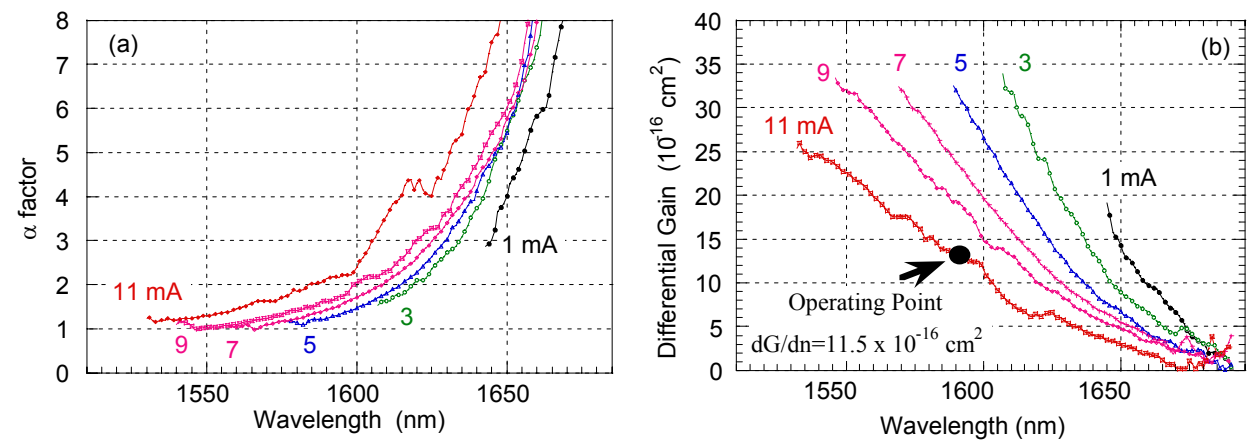

Fig. 7.1. The linewidth enhancement factor (a) and differential gain (b) versus wavelength at various bias currents.

This method is now commonly used for measuring the linewidth enhancement factor $\alpha_{T}$ [86, 87, 88]. P.Morton has shown how these measurements together with the gain measurements and carrier lifetime measurements [12] allow for optimization of the laser structure for high-speed operation [14]. Presented in the Fig. 7.1 are the measured dependencies of linewidth enhancement factor and differential gain on the wavelength for various currents. Optimizing the DFB wavelength from the data shown in Fig. 7.1 allowed achieving the small-signal direct modulation bandwidth of $26 \mathrm{GHz}$ and linewidth enhancement factor of near 1 .

Shown in the Fig. 7.1b is the set of the differential gain spectra determined using the below threshold gain spectra combined with the carrier lifetime measurements discussed in Sections 2.1 and 5.1 respectively. It should be pointed out that the value of the differential gain obtained using this method (indicated by solid circle in the Fig. 7.1b) is in very good agreement with the value of the differential gain obtained in the same device from the response measurements described in the Section 6.1 [14].

An alternative measurement technique for determination of $\alpha_{T}$ utilizes the optical gain spectra determined from TSE as described in Section 2.2. C. Henry [4] has used the Kramers-Krönig relation for small changes of refractive index: 


$$
\delta N^{\prime}=\frac{2}{\pi} P \int_{0}^{\infty} \frac{\delta N^{\prime \prime} \cdot \varepsilon^{\prime}}{\varepsilon^{\prime 2}-\varepsilon^{2}} \cdot d \varepsilon^{\prime}
$$

where $P$ indicates taking the principal part integral. Eq. (7.3) can be used to relate $\delta G$ and $\delta N^{\prime \prime}$ :

$$
\delta N^{\prime}=-\frac{h c}{2 \pi^{2}} P \int_{0}^{\infty} \frac{\delta G}{\varepsilon^{\prime 2}-\varepsilon^{2}} \cdot d \varepsilon^{\prime}
$$

The coefficient $\alpha_{T}$ can be determined by substituting Eq. (7.7) into Eq. (7.1). After some simple algebra:

$$
\alpha_{T}(\varepsilon)=\frac{\left.\frac{\partial N^{\prime}}{\partial \mu}\right|_{T}}{\left.\frac{\partial N^{\prime \prime}}{\partial \mu}\right|_{T}}=\frac{2}{\pi} \frac{\left.\varepsilon \cdot P \int_{0}^{\infty} \delta G\left(\varepsilon^{\prime}\right)\right|_{T} \frac{d \varepsilon^{\prime}}{\varepsilon^{\prime 2}-\varepsilon^{2}}}{\left.\delta G(\varepsilon)\right|_{T}}
$$

Equation (7.8) allows for determination of $\alpha_{T}$ from the gain spectrum. In order to perform this procedure, the gain spectra should be obtained in a very broad energy range. However, the commonly used Hakki-Paoli technique [8] for extraction of the gain spectra from amplified spontaneous emission (ASE) from the laser facet does not allow for that. Equation (7.8) requires some caution in treating the singularity in the integrand at $\varepsilon^{\prime}=\varepsilon$, procedure was described by C. Henry [4]. It should also be noticed, that the TSE intensity can be measured in arbitrary units because only the ratio of the integral of the spontaneous emission to its value at certain energy enters Eq. (7.8).

In [21] the results of the linewidth enhancement factor measurements in the DFB using both ASE and TSE spectra were reported. Obtained values are $\alpha_{T} \approx 2.0 \pm 0.2$ (using ASE measurements) and $\alpha_{T} \approx 2.1 \pm 0.2$ (using TSE measurements), which agree very well. The ASE measurements are usually easier to perform and more accurate, but sometimes the TSE type of the measurements are the only available solution.

Both methods described in this section are based on the below threshold measurements, and therefore can give only asymptotic value of the parameters close to threshold. However they have an advantage, since they can be used to study the dependence of the linewidth enhancement factor on carrier concentration and other parameters that cannot be varied in the real operating conditions above threshold. On the other hand the results of the above threshold measurements [82, 84] are more accurate since they can be obtained in the exact operating conditions of the device.

\section{Measurements of the carrier temperature and carrier heating in semiconductor lasers}

Typical semiconductor lasers operate at the injection current densities of an order of $10 \mathrm{kA} / \mathrm{cm}^{2}$ or higher. Under these conditions one can expect a significant difference between the lattice temperature $T_{L}$ and the temperature of the electron-hole-plasma in the active region $T_{e-h}$. This difference is determined by the power acquired by carriers in the active layer and their energy relaxation time. Both quantities have been disputed in the literature (see [89] and references there). 
Since the modal optical gain, wavelength chirp and carrier leakage over the heterobarrier are sensitive to the carrier temperature $T_{e-h}$, the study of carrier heating is important for understanding the laser physics and improving device design. Experimental studies of the heating in heterostructures using optical excitation and time resolved spectroscopy were carried out by different groups (see for example the review [90]). In semiconductor lasers and light-emitting diodes the carrier temperature is often estimated from the high-energy tail of the spontaneous emission spectra [91, 92, 93, 94]. Carrier temperatures up to $400 \mathrm{~K}$ at room temperature were reported in InGaAsP LED's [91]. Authors in [92] studied InGaAsP/InP buried heterostructure lasers with a bulk active layer. They did not observe carrier heating at room temperature for current densities up to $10 \mathrm{kA} / \mathrm{cm}^{2}$ (within an experimental accuracy of 10K). Authors in [93] studied photoexcieted InGaAsP/InP broad area quantum-well structures and found considerable heating at low temperatures. In AlInAs/GaInAs/InP bulk double heterostructure lightemitting transistors significant carrier heating at cryogenic temperatures was determined from the spontaneous emission profile [94] as well as using an analysis of thermionic emission [95].

The problem with the estimation of carrier temperature from the high energy tail of the spontaneous emission arises from the fact that the density of states as well as matrix element of the optical transition are not well known functions of energy. This makes it difficult to analyze the spontaneous emission (see for example Fig. 2.2a). Study of the thermionic emission is also nontrivial since it requires special sample geometry and is generally unsuitable for real laser structures.

\subsection{Determination of carrier heating from wavelength chirp and TSE measurements}

Recently a new experimental technique was proposed, which allows to measure the rate of change of the carrier plasma temperature with pumping current above threshold [21]. This method is based on the relationship between the rate of carrier heating and the wavelength chirp. Wavelength chirp results from the dependence of the real part of refractive index on current. The real and imaginary parts of refractive index are related through the Kramers-Krönig relation. Under lasing condition the distortion of spectral profile of the optical gain (and therefore the imaginary part of refractive index) is caused by spectral hole burning [54, 96, 97] and carrier heating [97, 98]. However, the experimental studies of fast dynamics of the optical gain in semiconductor laser structures have shown that the electron-hole plasma thermalization time is much shorter than the time needed for thermal equilibrium between the plasma and crystal lattice [99]. Therefore in the treatment of [21] the carrier heating was considered to be dominant and the spectral hole burning effect was neglected. In this case the energy distribution of electrons and holes is described by Fermi functions with the same temperature. This temperature differs from the lattice temperature; also electrons and holes have different quasi Fermi level energies when voltage is applied to a laser diode. Sources of carrier heating above threshold are injection of energetic carriers from heterobarriers into active layer and free-carrier absorption. The first effect depends on the injection current and the second on the optical field. Therefore to first order approximation both effects are proportional to the difference between operating and threshold currents.

In a static case above threshold the optical gain is equal to the optical loss. At the lasing wavelength, the reduction of the optical gain due to carrier heating is compensated 
by of the increase of the carrier concentration. Expressing this in terms of variations of the imaginary part of the refractive index yields:

$$
\left.\frac{\partial N^{\prime \prime}}{\partial \mu}\right|_{T} d \mu+\left.\frac{\partial N^{\prime \prime}}{\partial T}\right|_{\mu} d T=0
$$

However, pinning of the optical gain does not occur at all wavelengths. The real part of the refractive index $N^{\prime}$ is related to the imaginary part $N^{\prime \prime}$ (and optical gain) via the Kramers-Krönig relation. This results in the dependence of the refractive index on current above threshold and in the wavelength chirp. The full change of the real part of the index $N^{\prime}$ due to the same small variations $d \mu$ and $d T$, can be expressed in the same terms as in Eq. (8.1):

$$
\left.\frac{\partial N^{\prime}}{\partial \mu}\right|_{T} d \mu+\left.\frac{\partial N^{\prime}}{\partial T}\right|_{\mu} d T=d N^{\prime}
$$

The linewidth enhancement factor as defined in Eq. (7.1) can also be defined as a derivative with respect to the quasi Fermi level separation because only two variables out of three $(n, \mu$ and $T)$ are independent for Fermi-distributed carriers:

$$
\alpha_{T}=\frac{\left.\frac{\partial N^{\prime}}{\partial \mu}\right|_{T}}{\left.\frac{\partial N^{\prime \prime}}{\partial \mu}\right|_{T}}
$$

This definition allows for easier measurement, because quasi Fermi level separation $\mu$ is easier to determine than the carrier concentration.

The Kramers-Krönig relation suggests that there is an analogous parameter relating small variations of the real and imaginary parts of the refractive index due to the change of the carrier temperature at a fixed value of the quasi Fermi level separation:

$$
\alpha_{\mu}=\left.\frac{\left.\frac{\partial N^{\prime}}{\partial T}\right|_{\mu}}{\frac{\partial N^{\prime \prime}}{\partial T}}\right|_{\mu}=\left.\frac{\partial N^{\prime}}{\partial N^{\prime \prime}}\right|_{\mu}
$$

This definition is very close to one by C. Hultgren and E. Ippen [100]. It should be noted that unlike $\alpha_{T}$, the parameter $\alpha_{\mu}$ could be determined only from TSE measurements using a formula similar to Eq. (7.8). Determination of this parameter from ASE measurements is impossible because the shift of Fabry-Perot peaks with ambient temperature is determined mainly by the change of the effective index due to the change of the crystal lattice temperature, and not the carrier plasma temperature.

Combining Eqs. (8.1)-(8.4) one can relate the change of the real part of the refractive index and change of the carrier temperature:

$$
d N^{\prime}=-\left.\left(\alpha_{T}-\alpha_{\mu}\right) \frac{\partial N^{\prime \prime}}{\partial T}\right|_{\mu} \cdot d T
$$


Eq. (8.5) may be rewritten in terms of laser parameters that can be measured experimentally. Eqs. (7.3) and (7.4) can be used to express the change of the lasing wavelength due to the change of the carrier temperature in the active layer:

$$
d \lambda=\left.\frac{\lambda^{2}}{4 \pi \cdot N_{e f f}}\left(\alpha_{T}-\alpha_{\mu}\right) \cdot \frac{\partial g}{\partial T}\right|_{\mu} \cdot d T
$$

Carrier heating lifts the pinned carrier concentration (and quasi Fermi level separation) above threshold and results in wavelength chirp. The carrier heating affects the lasing wavelength in two ways, described by the two terms in brackets $\left(\alpha_{T}-\alpha_{\mu}\right)$ in Eq.(8.6). The first term corresponds to the change of the real part of the refractive index due to change of the quasi Fermi level separation. The second term describes the change in real part of the refractive index due to the change of the gain profile produced by the carrier heating.

To determine accurately the change of wavelength with current in a small-signal approach, one can use a Fabry-Perot etalon method or a gated delayed self-homodyne technique [101]. Rewriting the Eq. (8.6) for frequency chirp due to current modulation:

$$
\frac{d T}{d I}=-\frac{4 \pi \cdot N_{e f f}}{\left(\alpha_{T}-\alpha_{\mu}\right) \cdot c} \frac{1}{\left.\frac{\partial g}{\partial T}\right|_{\mu}} \beta
$$

where $\beta=\frac{d v}{d I}$ is the chirp parameter.

Eq. (8.7) establishes the relation between the rate of carrier heating above threshold and wavelength chirp. Coefficients $\left.\frac{\partial g}{\partial T}\right|_{\mu}, \alpha_{T}$ and $\alpha_{\mu}$ can be determined from the gain measurements described in previous sections. Their values were: $\left.\frac{\partial g}{\partial T_{e-h}}\right|_{\mu} \approx-0.45 \pm 0.05 \frac{\mathrm{cm}^{-1}}{\mathrm{~K}}, \alpha_{T} \approx 2.1 \pm 0.2, \alpha_{\mu} \approx-1.4 \pm 0.3$, and $\beta=156 \mathrm{MHz} / \mathrm{mA}$

[21]. Using these values, the rate of change of the carrier temperature with current was estimated to be approximately $0.13 \mathrm{~K} / \mathrm{mA}$. The accuracy of the estimation is about $25 \%$. This allows for an estimation of the carrier temperature difference between a threshold of $15 \mathrm{~mA}$ and a typical operating current of $50 \mathrm{~mA}$ to be $4.7 \mathrm{~K}$, which is consistent with the results of C. Henry et al [92]. This is considerably less than estimated by other authors (e.g. [102]) where the energy relaxation time of the electron-hole plasma was considered to be few picoseconds. The results of [21] suggest a subpicosecond energy relaxation time, which is consistent with the results of pump-probe experiments [99].

\section{Acknowledgments}

The authors acknowledge C. L. Reynolds and L. Shterengas for useful discussion, and T. Aherne for correcting errors. G. L. Belenky thanks ARO for support under grant DAAG55-97-1-0009. 


\section{References}

1. H. Casey and M. Panish, "Heterostructure Lasers", Academic Press, New York, 1978.

2. L. C. Coldren and S. W. Corzine, "Diode Lasers and Photonic Integrated Circuits", in Wiley Series in Microwave and Optical Engineering, John Wiley \& Sons, New York, 1995.

3. A. Yariv, "Optical Electronics", $4^{\text {th }}$ Edition, Saunders College Publishing, New York, 1991.

4. C. H. Henry, R. A. Logan, and K. A. Bartness, "Spectral dependence of the change in refractive index due to carrier injection in GaAs lasers", J. Appl. Phys. 52 (1981) 4457-4461.

5. A. Oster, G. Erbert, and H. Wenzel, "Gain spectra measurements by a variable stripe length method with current injection", Electron. Lett. 33 (1997) 864-886.

6. J. L. Zilko, L. J. P. Ketelsen, Y. Twu, D. P. Wilt, S. G. Napholtz, J. P. Blaha, K. E. Strege, V. G. Riggs, D. V. Vanharen, S. Y. Leung, P. M. Nitzsche, J. A. Long, G. Przyblek, J. Lopata, M. W. Focht, and L. A. Koszi, "Growth and characterization of high yield, reliable, high power, high-speed InP/InGaAsP capped mesa buried heterostructure distributed feedback lasers", IEEE J. Quantum Electron. QE-25 (1989) 2091-2095.

7. E. I. Gordon, "Optical Master Oscillators and Noise", The Bell System Technical Journal. (1964) 507-539.

8. B. W. Hakki and T. L. Paoli, "Gain spectra in GaAs double-heterojunction injection lasers", $J$. Appl. Phys. 46 (1975) 1299-1306.

9. D. T. Cassidy, "Technique for measurement of the gain spectra of semiconductor lasers", $J$. Appl. Phys. 56 (1984) 3096-3099.

10. L. A. Lam Sin Cho, P. M. Smowton, B. Thomas, "Spectral gain measurements for semiconductor laser diodes", IEE Proc. Part J. 137 (1990) 64-68.

11. G. E. Shtengel and D. A. Ackerman, "Internal optical loss measurements in $1.3 \mu \mathrm{m} \mathrm{InGaAsP}$ lasers", Electron.Lett. 31 (1995) 1157-1159.

12. G. E. Shtengel, D. A. Ackerman, P. A. Morton, E. J. Flynn, and M. S. Hybertsen, "Impedancecorrected carrier lifetime measurements in semiconductor lasers", Appl. Phys. Lett. 67 (1995) 1506-1508.

13. D. A. Ackerman, G. E. Shtengel, M. S. Hybertsen, P. A. Morton, R. F. Kazarinov, T. TanbunEk, and R. A. Logan, "Analysis of Gain in Determining $\mathrm{T}_{0}$ in $1.3 \mu \mathrm{m}$ Semiconductor Lasers", IEEE J. of Sel. Top. in Quantum Electron. 1 (1995) 250-263.

14. P. A. Morton, D. A. Ackerman, G. E. Shtengel, R. F. Kazarinov, M. S. Hybertson, T. TabunEk, R. A. Logan, A. M. Sergent, "Material Characteristics of $1.55 \mu \mathrm{m}$ High-Speed MQW Lasers", IEEE Photon. Techn. Lett. 7 (1995) 833-835.

15. V. Kozlov, A. Salokatve, A. V. Nurmikko, D. C. Grillo, Li He, J. Han, Y. Fan, M. Ringle, and R. L. Gunshor, "Gain characteristics of blue/green II-VI quantum well diode lasers", Appl. Phys. Lett. 65 (1994) 1863-1864.

16. C. Sirtori, J. Faist, F. Capasso, D. L. Sivco, A. L. Hutchinson, A. Y. Cho, "Pulsed and Continuous-Wave Operation of Long Wavelength Infrared $(\lambda=9.3 \mu \mathrm{m})$ Quantum Cascade Lasers", IEEE J. Quantum Electron. 33 (1997) 89-93.

17. V. P. Gribkovskii and V. A. Samoilyukovich, "Determination of the amplification coefficient, luminescence power, and separation between Fermi quasilevels in injection lasers", Zhurnal prikladnoi spektroskopii. 11 (1969) 170-172.

18. C. H. Henry, R. A. Logan, and F. R. Merrit, "Measurement of gain and absorption spectra in AlGaAs buried heterostructure lasers", J. Appl. Phys. 51 (1980) 3042-3050.

19. P. Blood, A. I. Kucharska, J. P. Jacobs, and K. Griffiths, "Measurement and calculation of spontaneous recombination current and optical gain in GaAs-AlGaAs quantum-well structures", J. Appl. Phys. 70 (1991) 1144-1156.

20. P. Rees and P. Blood, "Derivation of Gain Spectra of Laser Diodes from Spontaneous Emission Measurements”, IEEE J. Quantum Electron. 31 (1995) 1047-1050.

21. G. E. Shtengel, R. F. Kazarinov, G. L. Belenky, and C. L. Reynolds, Jr., "Wavelength Chirp and Dependence of Carrier Temperature on Current in MQW InGaAsP Lasers", IEEE J. Quantum Electron. 33 (1997) 1396-1402. 
22. K. L. Shaklee and R. F. Leheny, "Direct determination of optical gain in semiconductors", Appl. Phys. Lett. 18 (1971) 475-477.

23. P. S. Gross and W. G. Oldham, "Theory of optical gain measurements", IEEE J. Quantum Electron. QE-11 (1975) 190-197.

24. O. Hildebrand, E. Goebel and K. Loehnert, "Investigations on unsaturated optical gain spectra of GaAs/GaAllAs DHS lasers", J. Appl. Phys. 15 (1978) 149-152.

25. R. O. Miles, M. A. Dupertuis, F. K. Reinhart and P. M. Brosson, "Gain measurements in InGaAs/InGaAsP multiquantum-well broad area lasers", IEE Proc. Part J. 139 (1992) 33-38.

26. D. J. Bossert and D. Gallant, "Improved method for gain/index measurements of semiconductor lasers", Electron. Lett. 32 (1996) 338-339.

27. D. J. Bossert and D. Gallant, "Gain, refractive index and $\alpha$-parameter in InGaAs-GaAs SQW broad-area lasers",IEEE Photon. Techn. Lett. 8 (1996) 322-324.

28. D. V. Donetsky, G. L. Belenky, D. Z. Garbuzov, H. Lee, R. U. Martinelly, G. Taylor, S. Luryi, J. C. Connolly, "Direct measurements of heterobarrier leakage current and modal gain in 2.3 $\mu \mathrm{m}$ double QW p-substrate InGaAsSb/AlGaAsSb broad area lasers", accepted IEE Electron. Lett. 35 (1999).

29. C. H. Henty, R. A. Logan, F. R. Merritt, and J. P. Luongo, "The Efffect of the Intervalence Band Absorption on the Thermal Behavior of InGaAsP Lasers", IEEE J. Quantum Electron. QE-19 (1983) 947-952.

30. J. R. Biard, W. N. Carr, and B. S. Reed, "Analysis of a GaAs Laser", Trans. Metallurg. Soc. AIME, 230 (1964) 286-290.

31. H. C. Casey Jr., M. B. Panish, W. O. Schlosser, and T. L. Paoli, "GaAs-AlxGa1-xAs heterostructure laser with separate optical and carrier confinement”, J. Appl. Phys. 45 (1974) 322-333.

32. P. M. Smowton and P. Blood, "On the determination of internal optical mode loss of semiconductor lasers", Appl. Phys. Lett. 70 (1997) 2365-2367.

33. P. A. Andrekson, N. A. Olsson, T. Tanbun-Ek, R. A. Logan, D . L. Coblentz, and H.Temkin, "Novel technique for determining internal loss of individual semiconductor laser", Electron.Lett. 28 (1992) 171-172.

34. E. A. Avrutin, I. E. Chebunina, I. A. Eliashevitch, S. A. Gurevich, and G. E. Shtengel, "TE and TM optical gains in AlGaAs/GaAs single-quantum-well lasers", Semicond. Sci. Technol. 8 (1993) 80-87.

35. L. J. P. Ketelsen, "Simple technique for measuring cavity loss in semiconductor lasers", Electron.Lett. 30 (1994) 1422-1424.

36. V. V. Luc, P. G. Eliseev, M. A. Manko, and G. T. Mikaelian, "Electrical Diagnostics of the Amplifier Operation and a Feasibility of Signal Registration on the Basis of the Voltage Saturation Effect in Junction Laser Diodes", IEEE J. Quantum Electron. QE-19 (1983) 10801083.

37. A. G. P. Agrawal and N. K. Dutta, "Semiconductor lasers", Van Nostrand Reinhold, New York, 1993, p.127.

38. H. Yamazaki M. Yamaguchi and M. Kitamura, "Spectral linewidth rebroadening in MQWDFB LDs caused by spontaneous emission noise in SCH/barrier layers", IEEE Photon. Techn. Lett. 6 (1994) 341-343.

39. H. Ishikawa and I. Suemune, "Analysis of temperature dependent optical gain of strained quantum well taking account of carriers in SCH layer", IEEE Photon. Techn. Lett. 6 (1994) 344-347.

40. M. Buijs, K. Shahzad, S. Flamholtz, K. Haberen and J. Gaines, "Carrier leakage in blue-green II-VI semiconductor lasers", Appl. Phys. Lett. 67 (1995) 1987-1989.

41. B. Lane, D. Wu, H. J. Yi, J. Diaz, A. Rybaltowski, S. Kim, M. Erdtmann, H. Jeon and M. Razeghi, "Study on the effect of minority carrier leakage in InAsSb/InPAsSb double heterostructure", Appl. Phys. Lett. 70 (1997) 1447-1449.

42. Liang-Hui Chen, J. C. V. Mattos, F. C. Prince and N. B. Patel, "Spatially resolved observation of carrier leakage in 1.3- $\mu \mathrm{m} \operatorname{In}_{1-\mathrm{x}} \mathrm{Ga}_{\mathrm{x}} \mathrm{As}_{\mathrm{y}} \mathrm{P}_{1-\mathrm{y}}$ lasers", Appl. Phys. Lett. 44 (1984) 520-522. 
43. S. Yamakoshi, T. Sanada, O. Wada, I. Umebu and T. Sakurai, "Direct observation of electron leakage in InGaAsP/InP double heterostructure", Appl. Phys. Lett. 40 (1982) 144-146.

44. A. A. Bernussi, H. Temkin, D. L. Coblentz, R. A. Logan, "Effect of barrier recombination on the high temperature performance of quaternary multiquantum well lasers", Appl. Phys. Lett. 66 (1995) 67-69.

45. D. Garbuzov, G. -J. Shiau, V. Bulovic, M. Boroditsky, C. -P. Chao and S. R. Forrest, "Photoluminescence study of excess carrier spillover in $1.3 \mu \mathrm{m}$ wavelength strained multiquantum-well InGaAsP/InP laser structures”, Appl. Phys. Lett. 66 (1995) 1307-1309.

46. E. J. Flynn and D. A. Ackerman, "Electron leakage and the excess voltage at p-P heterojunctions in InGaAsP/InP lasers", IEDM 1997, 15.3.1, p.389.

47. T. R. Chen, S. Margalit, U. Koren, K. L. Yu, L. C. Chiu, A. Hasson and A. Yariv, "Direct measurement of the carrier leakage in an InGaAsP/InP laser", Appl. Phys. Lett. 42 (1983) 1000-1002.

48. G. L. Belenky, R. F. Kazarinov, J. Lopata, S. Luryi, T. Tanbun-Elk and P. A. Garbinski. "Direct measurement of the carrier leakage out of the active region in InGaAsP/InP laser heterostructures", IEEE Trans. Elec. Dev. 42 (1995) 215-218.

49. G. L. Belenky, C. L. Reynolds, D. V. Donetsky, G. E. Shtengel, M. Hybertsen, M. A. Alam, G. A. Baraff, R. K. Smith, R. F. Kazarinov, J. Winn, L. E. Smith, "Role of p-doping profile and regrowth on the static characteristics of $1.3 \mu \mathrm{m} \mathrm{MQW} \mathrm{InGaAsP/InP} \mathrm{lasers.} \mathrm{Experiment}$ and modeling", submitted to IEEE J. Quantum Electron. (1999).

50. G. L. Belenky, D. V. Donetsky, C. L. Reynolds, Jr., R. F. Kazarinov, G. E. Shtengel, S. Luryi, J. Lopata, "Temperature performance of $1.3 \mu \mathrm{m}$ InGaAsP-InP lasers with different profile of p-doping”, IEEE Photon. Techn. Lett. 9 (1997) 1558-1560.

51. R. F. Kazarinov and M. R. Pinto, "Carrier transport in laser heterostructures", IEEE J. Quantum Electron. 30 (1994) 49-53.

52. H. Statz and G. deMars, in "Quantum Electronics" (ed. C. H. Towns), Columbia Univ.Press, New York, 1960, p.235.

53. D. A. Kleinman, Bell. Syst. Tech. J. 43 (1964) 1505.

54. T. L. Koch and R. A. Linke, "Effect of nonlinear gain reduction on semiconductor laser wavelength chirping", Appl. Phys. Lett. 48 (1986) 613-615.

55. R. Nagarajan, M. Ishikawa, T. Fukushima, R. S. Geels, and J. E. Bowers, "High Speed Quantum-Well Lasers and Carrier Transport Effects", IEEE J. Quantum Electron. QE-28 (1992) 1990-2008.

56. S. Weisser, I. Esquivias, P. J. Tasker, J. D. Ralston, B. Romero and J.Rosezweig, "Impedance Characteristics of Quantum-Well Lasers", IEEE Photon. Tech. Lett. 6 (1994) 1421-1423.

57. I. Esquivias, S. Weisser, B. Romero, J. D. Ralston, and J.Rosezweig, "Carrier Capture and Escape times in $\mathrm{In}_{0.35} \mathrm{Ga}_{0.65} \mathrm{As}-\mathrm{GaAs}$ Multiquantum-Well Lasers Determined from HighFrequency Electrical Impedance Measurements", IEEE Photon. Techn. Lett. 8 (1996) 12941296.

58. R. Olshansky, C. B. Su, J. Manning and W. Powazinik, "Measurement of Radiative and Nonradiative Recombination Rates in InGaAsP and AlGaAs Light Sources", IEEE J. Quantum Electron. QE-20 (1984) 838-854.

59. R. Olshansky, J. LaCourse, T. Chow and W. Powazinik, "Measurement of Radiative, Auger, and Nonradiative Currents in $1.3 \mu \mathrm{m}$ InGaAsP Buried Heterostructure Lasers", Appl. Phys. Lett. 50 (1987) 310-312.

60. G. H. B. Thompson, "Analysis of radiative and nonradiative recombination law in lightly doped InGaAsP Lasers", Electron. Lett. 19 (1983) 154-155.

61. K. Konnerth and C. Lanza, "Delay between current pulse and light emission of a gallium arsenide injection laser", Appl. Phys. Lett. 4 (1964) 120-121.

62. Y. Zou, J. S. Osinski, P. Godzinski, P. D. Dapkus, W. C. Rideout, W. F. Sharfin, J. Schlafer and F. D. Crawford, "Experimental Study of $1.55 \mu \mathrm{m}$ Compressively Strained Semiconductor Lasers", IEEE J. Quantum Electron. QE-29 (1993) 1565-1575.

63. J. Katz, S. Margalit, C. Harder, D. Wilt and A.Yariv, "The Intrinsic Equivalent Circuit of a Laser Diode”, IEEE J. Quantum Electron. QE-17 (1981) 4-7. 
64. R. S. Tucker, "Circuit model of double-heterojunction laser below threshold", IEE Proc. Part I. 128 (1981) 101-106.

65. E. J. Flynn, "Carrier-Density-Independent Radiative Constant in $1.3 \mu \mathrm{m}$ Buried Heterostructure Lasers", J. Appl. Phys. 78 (1995) 4046-4052.

66. G. E. Shtengel, D. A. Ackerman, P. A. Morton, "True carrier lifetime measurements of semiconductor laser", Electron. Lett. 31 (1995) 1747-1748.

67. M. Morishita, T. Ohmi and J. Nishizawa, "Impedance characteristics of double-heterostructure laser diodes", Solid-State Electron. 22 (1979) 951-962.

68. W. B. Joyce and R. W. Dixon, "Electrical characterization of heterostructure lasers", J. Appl. Phys. 49 (1978) 3719-3728.

69. S. C. Kan and K. Y. Lau, "Intrinsic Equivalent Circuit of Quantum-Well Lasers", IEEE Photon. Techn. Lett. 4 (1992) 528-530.

70. R. S. Tucker, "High-speed modulation of semiconductor lasers", J. Lightwave Technol. LT-3 (1985) 1180-1192.

71. J. E. Bowers, "High-speed semiconductor laser design and performance", Solid State Electron. 30 (1987) 1-11.

72. T. Fukushima, R. Nagarajan, M. Ishikawa and J. E. Bowers, "High-speed dynamics in InP based multiple quantum well lasers", Jpn. J. Appl. Phys. 32 (1993) 70-83.

73. R. Olshansky, P. Hill, V. Lanisera, and W. Powazinik, "Frequency Response of $1.3 \mu \mathrm{m}$ InGaAsP, High Speed Semiconductor Lasers", IEEE J. Quantum Electron. QE-23 (1987) $1410-1418$.

74. P. A. Morton, T. Tanbun-Ek, R. A. Logan, A. M. Sergent, P. F. Sciortino, Jr., and D. L. Coblentz, "Frequency Response Substraction for Simple Measurement of Intrinsic Laser Dynamic Properties", IEEE Photon. Techn. Lett. 4 (1992) 133-136.

75. G. L. Belenky, C. L. Reynolds, L. Shterengas, D. V. Donetsky, G. E. Shtengel, M. Hybertsen, R. F. Kazarinov, S. Luryi, "High -Speed Dynamics in $1.3 \mu \mathrm{m}$ InGaAsP/InP Multiple Quantum Well DFB Lasers with Different p-Doping Profiles", unpublished.

76. L. D. Westbrook, N. C. Fletcher, D. M. Cooper, M. Stevenson, P. C. Spurdens, "Intensity noise in $1.55 \mu \mathrm{m}$ GaInAs quantum well buried heterostructure lasers", Electron. Lett. 25 (1989) 1183-1184.

77. M. C. Tatham, I. F. Lealman, C. P. Seltzer, L. D. Westbrook, D. M. Cooper, "Resonance frequency, damping and differential gain in $1.55 \mu \mathrm{m}$ multiple quantum-well lasers", IEEE $J$. Quantum Electron. 28 (1992) 408-414.

78. H. Lu, G. P. Li, T. Makino, "High-speed performance of partly gain-coupled 1.55- $\mu \mathrm{m}$ strained layer multiple-quantum-well DFB lasers", IEEE Photon. Techn. Lett. 8 (1993) 861-863.

79. A. Wakatsuki, Y. Kawamura, Y. Noguchi, H. Iwamura, "Effect of conduction-band discontinuity on lasing characteristics of $1.5 \mu \mathrm{m} \mathrm{InGaAs/In(Ga)AlAs} \mathrm{MQW-FP} \mathrm{lasers",} \mathrm{IEEE}$ Photon. Techn. Lett. 4 (1993) 383-386.

80. M. W. Fleming and A. Mooradian, "Fundamental Line broadening of single mode GaAlAs diode lasers", Appl. Phys. Lett. 38 (1981) 511-513.

81. C. H. Henry, "Theory of the linewidth of semiconductor lasers", IEEE J. Quantum Electron. QE-18 (1982) 259-264.

82. K. Vahala and A. Yariv, "Semiclassical theory of noise in semiconductor lasers", IEEE J. Quantum. Electron. QE-19 (1983) 1096-1109.

83. C. Harder, K. Vahala, and A. Yariv, "Measurement of the linewidth enhancement factor $\alpha$ of semiconductor lasers", Appl. Phys. Lett. 42 (1983) 328-330.

84. F. Devaux, Y. Sorel, and J. F. Kerdilis, "Simple Measurement of Fiber Dispersion and of Chirp Parameter of Intensity Modulated Light Emitter", J. Light. Techn. 11 (1993) 1937-1940.

85. I. D. Henning and J. V. Collins, "Measurements of the Semiconductor Laser Linewidth Broadening Factor", Electron. Lett. 19 (1983) 927-929.

86. L. D. Westbrook, B. Eng, "Measurements of $d g / d N$ and $d n / d N$ and their dependence on photon energy in $\lambda=1.55 \mu \mathrm{m}$ InGaAsP laser diodes", IEE Proc. Part J. 133 (1986) 135-142. 
87. W. Rideout, B. Yu, J. LaCourse, P. K. York, K. J. Beernink, and J. J. Coleman, "Measurement of the carrier dependence of differential gain, refractive index, and linewidth enhancement factor in strained-layer quantum well lasers", Appl. Phys. Lett. 56 (1990) 706-708.

88. A. S. Schönfelder, S. Weisser, J. D. Ralston, J. Rosenzweig, "Differential Gain, Refractive Index, and Linewidth Enhancement Factor in High-Speed GaAs-Based MQW Lasers: Influence of Strain and p-Doping", IEEE Photon. Techn. Lett. 6 (1994) 891-893.

89. V. I. Tolstikhin and M. Willander, "Carrier Heating Effects in Dynamic-Single-Frequency GaInAsP-InP Laser Diodes", IEEE J. Quantum Electron. 31 (1995) 814-833.

90. J. Shah, "Photoexcieted hot carriers: from CW to $6 \mathrm{fs}$ in 20 years", Solid-State Electron. 32 (1989) 1051-1056.

91. J. Shah, R. F. Leheny, and R. E. Nahory, "Hot-carrier effects in $1.3 \mu \mathrm{m} \operatorname{In}_{1-\mathrm{x}} \mathrm{Ga}_{\mathrm{x}} \mathrm{As}_{\mathrm{y}} \mathrm{P}_{\mathrm{y}-1}$ light emitting diodes", Appl. Phys. Lett. 39 (1981) 618-620.

92. C. H. Henry, R. A. Logan, H. Temkin, and F. R. Merrit, "Absorption, Emission, and Gain Spectra in $1.3 \mu \mathrm{m}$ InGaAsP Quaternary Lasers", IEEE J. Quantum Electron. QE-19 (1983) 941-946.

93. D. Z. Garbuzov, V. P. Chalyi, A. E. Svelokuzov, V. B. Khalfin, and A. L. Ter-Martirosyan, "Auger recombination and heating of carriers at high rates of photoexcitation of InGaAsP/InP $(\lambda=1.3 \mu \mathrm{m})$ and $\mathrm{InGaAsP} / \mathrm{GaAs}(\lambda=0.85 \mu \mathrm{m})$ quantum-well heterostructures", Sov. Phys. Semicond. 22 (1988) 410-413.

94. G. L. Belenky, P. A. Garbinski, S. Luryi, M. Mastrapasqua, A. Y. Cho, R. A. Hamm, T. R. Hayes, E. J. Laskowski, D. L. Sivco, and P. R. Smith, "Collector-up-light-emitting charge injection transistors in $n$-InGaAs/InAlAs/ $p$-InGaAs and $n$-InGaAs/ $p$-InGaAs heterostructures", J. Appl. Phys. 73 (1993) 8618-8627.

95. G. L. Belenky, A. Kastalsky, S. Luryi, P. A. Garbinski, A. Y. Cho, and D. L. Sivco, "Measurement of the effective temperature of majority carriers under injection of hot minority carriers in heterostructures", Appl. Phys. Lett. 64 (1994) 2247-2249.

96. R. F. Kazarinov, C. H. Henry, and R. A. Logan, "Longitudinal mode self-stabilization in semiconductor lasers", J. Appl. Phys. 53 (1982) 4631-4644.

97. K. L. Hall, J. Mark, E. P. Ippen, and G. Eisenstein, "Femtosecond gain dynamics in InGaAsP optical amplifiers", Appl. Phys. Lett. 56 (1990) 1740-1742.

98. M. S. Stix, M. P. Kesler, and E. P. Ippen, "Observation of subpicosecond dynamics in GaAlAs laser diodes”, Appl. Phys. Lett. 48 (1986) 1722-1724.

99. K. L. Hall, G. Lenz, A. M. Darwish, and E. P. Ippen, "Subpicosecond gain and Index Nonlinearities in InGaAsP Diode Lasers", Opt. Comm. 111 (1994) 589-612.

100. C. T. Hultgren and E. P. Ippen, "Ultrafast refractive index dynamics in AlGaAs diode laser amplifiers", Appl. Phys. Lett. 59 (1991) 635-637.

101. D. M. Baney and W. V. Sorin, "Measurement of a modulated DFB laser spectrum using gated delayed self-homodyne technique", Electron. Lett. 24 (1988) 669-670.

102. V. D. Pishchalko and V. I. Tolstikhin, "Carrier heating effects in InGaAsP/InP injection heterolasers", Sov. Phys. Semicond. 24 (1990) 288-294. 